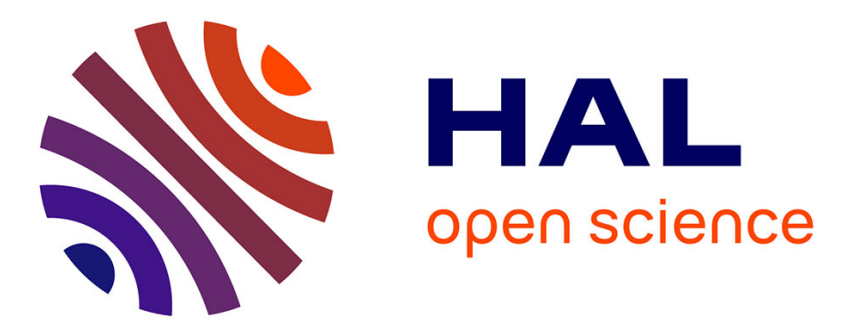

\title{
Optimal linear spatial filters for event-related potentials based on a spatio-temporal model: Asymptotical performance analysis
}

Bertrand Rivet, Antoine Souloumiac

\section{- To cite this version:}

Bertrand Rivet, Antoine Souloumiac. Optimal linear spatial filters for event-related potentials based on a spatio-temporal model: Asymptotical performance analysis. Signal Processing, 2013, 93 (2), pp.387-398. 10.1016/j.sigpro.2012.07.019 . hal-00742644

\section{HAL Id: hal-00742644 \\ https://hal.science/hal-00742644}

Submitted on 16 Oct 2012

HAL is a multi-disciplinary open access archive for the deposit and dissemination of scientific research documents, whether they are published or not. The documents may come from teaching and research institutions in France or abroad, or from public or private research centers.
L'archive ouverte pluridisciplinaire HAL, est destinée au dépôt et à la diffusion de documents scientifiques de niveau recherche, publiés ou non, émanant des établissements d'enseignement et de recherche français ou étrangers, des laboratoires publics ou privés. 


\title{
Optimal linear spatial filters for event-related potentials based on a spatio-temporal model: asymptotical performance analysis
}

\author{
Bertrand Rivet ${ }^{\mathrm{a}, *}$, Antoine Souloumiac ${ }^{\mathrm{b}}$ \\ ${ }^{a}$ GIPSA-lab, CNRS UMR-5216, 11, rue des Mathématiques, Campus Universitaire, Grenoble University, F-38402 Grenoble Cedex, France \\ ${ }^{b}$ CEA, LIST, Laboratoire Outils pour l'Analyse de Données, F-91191 Gif-Sur-Yvette, France
}

\begin{abstract}
In this paper, the estimation of spatio-temporal patterns in the context of event-related potentials or evoked potentials studies in neuroscience is addressed. The proposed framework (denoted xDAWN) has the advantage to require only the knowledge of the time of stimuli onsets which are determined by the experimental setup. A theoretical analysis of the xDAWN framework shows that it provides asymptotically optimal spatial filters under weak assumptions. The loss in signal to interference-plus-noise ratio due to finite sample effect is calculated in a closed form at the first order of perturbation and is then validated by simulations. This last result shows that the proposed method provides interesting performance and outperforms classical methods, such as independent component analysis, in a wide range of situations. Moreover, the xDAWN algorithm has the property to be robust with respect to the model parameter values. Finally, validations on real electro-encephalographic data confirm the good behavior of the proposed xDAWN framework in the context of a P300 speller brain-computer interface.
\end{abstract}

Keywords: biomedical signal processing, spatial filtering, asymptotical performance analysis, brain-computer interface, P300 speller

\section{Introduction}

In cognitive neuroscience, it is useful to explore brain activity through evoked potentials (EP) or event-related potentials (ERP) recorded by electro-encephalography (EEG), e.g. [1, 2]. For instance, ERPs allow to investigate i) the basic functional pathways through early ERPs or EPs as auditory, visual or somatosensory networks, and ii) cognitive pathways through late ERPs which are more related to memory tasks, execution of attention and emotion. ERP experiments usually involve the presentation of several kinds of stimuli and suppose that there exists a typical spatio-temporal pattern which is time-locked to each kind of stimuli (also called events).

In this context, EEG recorded signals do not only contain the spatio-temporal patterns linked to the events but also ongoing brain activity as well as muscular and/or ocular artifacts. As a consequence, to ease the estimation of such spatio-temporal patterns, one can repeat the experiments but this solution needs to record more data. This method is based on the assumption that the ERP waveforms are uncorrelated with the ongoing cerebral activity and with the artifacts: the ERP waveforms can thus be estimated by a straightforward or a weighted average of the

\footnotetext{
${ }^{*}$ Corresponding author.

Bertrand Rivet

GIPSA-lab

11 rue des mathématiques, Grenoble Campus

BP 46

F - 38402 SAINT MARTIN D'HERES Cedex.

Email addresses: bertrand.rivet@gipsa-lab.grenoble-inp.fr (Bertrand Rivet), antoine.souloumiac@cea.fr (Antoine Souloumiac)
}

trials temporally aligned to the stimuli onsets [3]. The main drawback of this approach is that it only exploits the temporal aspect of the ERP. Another typical way to improve these estimates is to enhance the ERPs by a spatial filtering of the channels. Several methods based on independent component analysis (ICA) [4-8] have thus been proposed to enhance the signalto-noise ratio (SNR) or to remove the artifacts, e.g., [9-11]. In addition, after the optimization stage, these methods need to select the components (manually or using spatio-temporal prior knowledge). However, these methods often fail to extract correctly the ERP component since in a real experiment, the ERP components have a very small amplitude (about $\mu V$ ) compared to ongoing cerebral activity (about $m V$ ) and to ocular artifacts (about $100 \mathrm{mV}$ ). These methods are mainly based on spatial assumptions and do not exploit the temporal structures of the ERPs.

To avoid such limitations, methods based on a spatiotemporal model have been developed. For instance, common spatial pattern (CSP) [12, 13] or Fisher's linear discriminant analysis (LDA) [14] are two classical methods to estimate spatial filters. CSP aims at simultaneously maximizing the power of one ERP and minimizing the power the other ERPs: it tries to maximize the signal-to-interference ratio (SIR). LDA is based on the maximization of the distance between two classes while it minimizes the variance within each class. More recently, several methods (e.g., [15-17]) investigate more complex spatiotemporal models. For instance in [16], a regular parametric waveform of the ERP is imposed to estimate the spatial filters. In [17], a direct estimation of the temporal waveform and the related spatial distribution without parameter selection 
has been proposed. However, all these methods are not able to deal with ERP waveforms that can temporally overlap each others with correlation, within one kind of ERPs and/or between several kinds of ERPs. In our previous studies [18, 19], the xDAWN algorithm has been introduced. It aims at estimating jointly the temporal signature and the spatial distribution of the ERPs, as well as the spatial filters that provide the largest signal-to-signal-plus-noise ratio (SSNR). The main advantage of this framework is its absence of assumptions either on the temporal waveform and the spatial distribution. The only prior knowledge is the onsets of the stimuli used in the experiment. In this contribution, a theoretical analysis of xDAWN framework is derived: it shows that the proposed method i) is asymptotically optimal and ii) has a good behavior, at the first order of perturbations, by substituting exact parameter values by estimated ones from the data. In addition, since no particular assumptions is imposed, the proposed xDAWN framework can be easily adopted for solving similar estimation problems if the proposed model is verified.

The rest of this paper is organized as follows. Section 2 summarizes the xDAWN framework. The theoretical analysis of its optimality and the asymptotical performance analysis are derived in Section 3. Section 4 investigates the links between xDAWN algorithm and other classical methods to estimate spatial filters in an ERP paradigm. Section 5 presents numerical experiments and validation on real EEG data, and Section 6 concludes this paper.

\section{XDAWN spatial filters}

In this section, the proposed xDAWN framework is briefly summarized.

\subsection{Model}

In the context of ERPs analysis, which supposes that there exists a typical spatio-temporal pattern time-locked with the stimuli, EEG signals $\mathbf{x}(k) \in \mathbb{R}^{N_{s}}$ recorded from $N_{s}$ sensors can be modeled as the superposition of the $N_{e}$ signals related to each of the $N_{e}$ classes of events (i.e. kinds of stimulations) and ongoing brain activity as well as ocular and/or muscular artifacts $\mathbf{n}(k) \in \mathbb{R}^{N_{s}}$. To take into account the variability of each ERP in a particular class that can appear during the experiment, one can assume that the $j$-th ERP of the $i$-th class, denoted $\mathbf{p}_{i, j}(k) \in \mathbb{R}^{N_{s}}$, is composed of a spatio-temporal pattern, $\mathbf{p}_{i}^{(c)}(k) \in \mathbb{R}^{N_{s}}$, common to all ERPs of the $i$-th class and of a random spatio-temporal pattern $\mathbf{p}_{i, j}^{(r)}(k) \in \mathbb{R}^{N_{s}}$ different for all ERPs of the $i$-th class:

$$
\mathbf{p}_{i, j}(k)=\mathbf{p}_{i}^{(c)}(k)+\mathbf{p}_{i, j}^{(r)}(k) .
$$

As a consequence, one can model the raw EEG as

$$
\mathbf{x}(k)=\sum_{i=1}^{N_{e}} \sum_{j=1}^{K_{i}} \mathbf{p}_{i, j}\left(k-\tau_{i}(j)\right)+\mathbf{n}(k),
$$

where $\tau_{i}(j)$ is the index time of the $j$-th stimulus of the $i$-th ERP class and $K_{i}$ is the number of stimuli of the $i$-th ERP class.
Basic algebraic manipulations lead to rewrite the convolutional model (1) in matrix notation as

$$
X=\sum_{i=1}^{N_{e}} \sum_{j=1}^{K_{i}} D_{i, j} P_{i, j}+N,
$$

where the $k$-th row of $X \in \mathbb{R}^{N_{t} \times N_{s}}$ (resp. $N$ ) is $\mathbf{x}(k)^{T}$ (resp. $\left.\mathbf{n}(k)^{T}\right)$ and $N_{t}$ is the total number of time samples. ${ }^{T}$ is the transpose operator. $P_{i, j} \in \mathbb{R}^{M_{i} \times N_{s}}$ is the $j$-th ERP spatiotemporal pattern of the $i$-th class of stimuli whose $k$-th row is $\mathbf{p}_{i, j}(k)^{T} . D_{i, j} \in \mathbb{R}^{N_{t} \times M_{i}}$ is a Toeplitz matrix whose first column entries are null but $D_{i, j}\left(\tau_{i}(j), 1\right)=1 . M_{i}$ is the number of time samples of the temporal pattern of $i$-th class of ERPs. In (2), $\sum_{j} D_{i, j} P_{i, j}$ thus models the signals related to the $i$-th class of events. Since $P_{i, j}$ is often a singular matrix (i.e. of reduced rank), spatio-temporal patterns can be factorized as $P_{i, j}=A_{i, j} W_{i, j}^{T}$, where $A_{i, j} \in \mathbb{R}^{M_{i} \times N_{s_{i}}}$ is temporal pattern of reduced dimensions and $W_{i, j} \in \mathbb{R}^{N_{s} \times N_{s_{i}}}$ is its spatial distribution over sensors, with $N_{s_{i}}<N_{s}$.

Moreover, one can assume that the differences between spatio-temporal patterns $P_{i, j}$ among the same class of ERP only come from temporal differences and not from spatial ones ${ }^{1}$ :

$$
P_{i, j}=\left(A_{i}^{(c)}+A_{i, j}^{(r)}\right) W_{i}^{T}
$$

where $A_{i}^{(c)} \in \mathbb{R}^{M_{i} \times N_{s_{i}}}$ denotes the common temporal pattern and $A_{i, j}^{(r)} \in \mathbb{R}^{M_{i} \times N_{s_{i}}}$ models the random temporal pattern. As a consequence, model (2) can be expressed as

$$
X=\sum_{i=1}^{N_{e}}\left(D_{i}^{(c)} A_{i}^{(c)}+D_{i}^{(r)} A_{i}^{(r)}\right) W_{i}^{T}+N
$$

where $D_{i}^{(c)}=\sum_{j=1}^{K_{i}} D_{i, j}, D_{i}^{(r)}=\left[D_{i, 1}, \cdots, D_{i, K_{i}}\right]$ and $A_{i}^{(r)}=$ $\left[A_{i, 1}^{(r)^{T}}, \ldots, A_{i, K_{i}}^{(r)}\right]^{T} \in \mathbb{R}^{\left(M_{i} K_{i}\right) \times N_{s_{i}}}$ are white centered Gaussian random variables.

In ERP analysis, one is generally only interested in the common (averaged) temporal patterns $A_{i}^{(c)}$.

\section{2. $x D A W N$ framework}

xDAWN algorithm aims at estimating $N_{f_{i}}$ spatial filters $U_{i} \in$ $\mathbb{R}^{N_{s} \times N_{f_{i}}}$ such that the SSNR of the $i$-th ERP after spatial filtering is maximized

$$
\tilde{U}_{i}=\arg \max _{U} \tilde{\rho}_{i}(U),
$$

where the SSNR is defined by

$$
\tilde{\rho}_{i}(U)=\frac{\operatorname{Tr}\left(U^{T} \tilde{\Sigma}_{i} U\right)}{\operatorname{Tr}\left(U^{T} \tilde{\Sigma}_{X} U\right)},
$$

with

$$
\begin{aligned}
\tilde{\Sigma}_{X} & =\frac{E\left[X^{T} X\right]}{N_{t}}, \\
\tilde{\Sigma}_{i} & =\frac{E\left[P_{i}^{(c)^{T}} D_{i}^{(c)^{T}} D_{i}^{(c)} P_{i}^{(c)}\right]}{N_{t}},
\end{aligned}
$$

${ }^{1}$ This is a reasonable assumption, since one can assume that the neurons involved in a specific cognitive task remain the same during the experiment while their temporal activity could be different. 
where $P_{i}^{(c)}=A_{i}^{(c)} W_{i}^{T}$. In practice since neither the actual spatio-temporal patterns $P_{i}^{(c)}$ and nor the actual durations of these patterns $M_{i}$ are known, xDAWN algorithm maximizes the estimated SSNR $\hat{\rho}_{i}(U)$

$$
\hat{U}_{i}=\arg \max _{U} \hat{\rho}_{i}(U)
$$

with

$$
\hat{\rho}_{i}(U)=\frac{\operatorname{Tr}\left(U^{T} \hat{\Sigma}_{i} U\right)}{\operatorname{Tr}\left(U^{T} \hat{\Sigma}_{X} U\right)},
$$

where the expectations in (6) and (7) are replaced by their stochastic estimates

$$
\begin{aligned}
\hat{\Sigma}_{X} & =\frac{X^{T} X}{N_{t}} \\
\hat{\Sigma}_{i} & =\frac{\hat{P}_{i}^{(c)^{T}} \hat{D}_{i}^{(c)^{T}} \hat{D}_{i}^{(c)} \hat{P}_{i}^{(c)}}{N_{t}} .
\end{aligned}
$$

Moreover, in the latter expression, $\hat{D}_{i}^{(c)}$ and $\hat{P}_{i}^{(c)}$ are estimates of these actual values. Like the actual matrix $D_{i}^{(c)}, \hat{D}_{i}^{(c)}$ is a Toeplitz matrix defined from the set of stimuli onsets $\tau_{i}(j)$ and the estimated durations of the ERP $\hat{M}_{i} . \hat{P}_{i}^{(c)}$ is estimated in the least mean square (LMS) sense by

$$
\hat{P}^{(c)} \triangleq \arg \min _{P}\left\|X-\hat{D}^{(c)} P\right\|_{F}^{2}=\left(\hat{D}^{(c)^{T}} \hat{D}^{(c)}\right)^{-1} \hat{D}^{(c)^{T}} X,
$$

where $\hat{D}^{(c)}=\left[\hat{D}_{1}^{(c)}, \cdots, \hat{D}_{N_{e}}^{(c)}\right]$ and $\hat{P}^{(c)}=\left[\hat{P}_{1}^{(c)^{T}}, \cdots, \hat{P}_{N_{e}}^{(c)^{T}}\right]^{T}$. Consequently,

$$
\hat{P}_{i}^{(c)}=\hat{B}_{i}^{T} X
$$

with $\hat{B}_{i}^{T}$ the matrix of corresponding rows in $\left(\hat{D}^{(c)^{T}} \hat{D}^{(c)}\right)^{-1} \hat{D}^{(c)^{T}}$. It is worth noting that $\hat{B}_{i}^{T}$ is different from $\left(\hat{D}_{i}^{(c)^{T}} \hat{D}_{i}^{(c)}\right)^{-1} \hat{D}_{i}^{(c)^{T}}$ as soon as assumed ERPs of the $i$-th class overlap assumed ERPs of at least one other class. The decomposition $\left(A_{i}^{(c)}, W_{i}\right)$ of the spatio-temporal pattern $P_{i}^{(c)}$ and the associated spatial filters which maximize the SSNR are given by the following theorem.

Theorem 1 (Estimations of $A_{i}^{(c)}, W_{i}$ and $U_{i}$ ). Let us define the generalized eigenvalue decomposition (GEVD) [20] of the $\operatorname{pair}\left(\hat{\Sigma}_{i}, \hat{\Sigma}_{X}\right)$ by

$$
\hat{\Sigma}_{i} \Theta_{i}=\hat{\Sigma}_{X} \Theta_{i} \Lambda_{i},
$$

where $\Lambda_{i}$ is the diagonal matrix of eigenvalues sorted in the descending order $\lambda_{1}^{(i)}>\cdots>\lambda_{N_{s}}^{(i)}$ and related eigenvectors $\Theta_{i}=$ $\left[\boldsymbol{\theta}_{1}^{(i)}, \cdots, \boldsymbol{\theta}_{N_{s}}^{(i)}\right]$ with $\Theta_{i}^{T} \hat{\Sigma}_{X} \Theta_{i}=I$, where I is the identity matrix.

The $N_{f}$ spatial filters $\hat{U}_{i}$ which satisfy (8) are given through the GEVD of the pair $\left(\hat{\Sigma}_{i}, \hat{\Sigma}_{X}\right)$ by the $N_{f}$ eigenvectors associated with the $N_{f}$ largest eigenvalues

$$
\hat{U}_{i}=\left[\theta_{1}^{(i)}, \cdots, \theta_{N_{f}}^{(i)}\right]
$$

Moreover, the spatial distribution is given by

$$
\hat{W}_{i}=\hat{\Sigma}_{X}\left[\theta_{1}^{(i)}, \cdots, \theta_{N_{f}}^{(i)}\right]
$$

and the temporal pattern is obtained from

$$
\hat{A}_{i}^{(c)}=\hat{B}_{i}^{T} X\left[\theta_{1}^{(i)}, \cdots, \theta_{N_{f}}^{(i)}\right]
$$

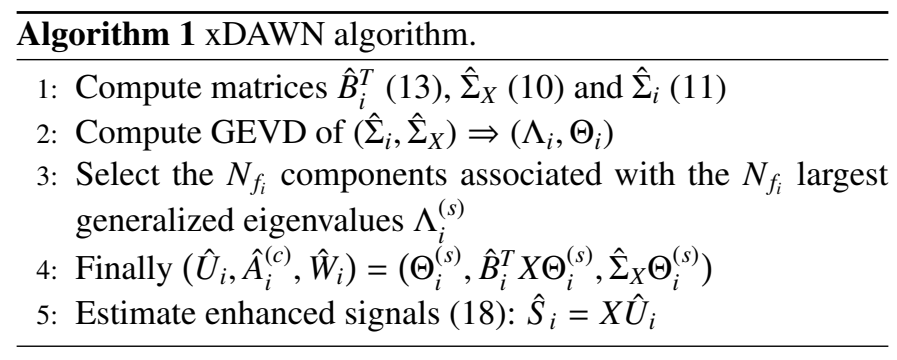

Proof Since (9) is a Rayleigh quotient of the pair $\left(\hat{\Sigma}_{i}, \hat{\Sigma}_{X}\right)$, it is straightforward to obtain (14). To obtain (15) and (16), one can rewrite criterion (8), using the QR decompositions [20] of $X$ and $D_{i}^{(c)}$, as

$$
\hat{V}_{i}=\arg \max _{V} \frac{\operatorname{Tr}\left(V^{T} Q_{X}^{T} \hat{B}_{i} R_{i}^{T} R_{i} \hat{B}_{i}^{T} Q_{X} V\right)}{\operatorname{Tr}\left(V^{T} V\right)},
$$

with $V=R_{X} U, X=Q_{X} R_{X}$ and $\hat{D}_{i}^{(c)}=Q_{i} R_{i}$, where $Q_{X}$ and $Q_{i}$ are orthogonal matrices, and $R_{X}$ and $R_{i}$ are upper triangular matrices, respectively. $\hat{V}_{i}$ are thus obtained by maximizing the Rayleigh quotient using the singular value decomposition (SVD) of matrix $R_{i} \hat{B}_{i}^{T} Q_{X}$ :

$$
R_{i} \hat{B}_{i}^{T} Q_{X}=\Phi_{i} \Delta_{i} \Psi_{i}^{T}
$$

where $\Phi_{i}$ and $\Psi_{i}$ are two unitary matrices and $\Delta_{i}$ is a diagonal matrix with nonnegative diagonal entries in decreasing order. As a consequence, one can rewrite $\hat{P}_{i}^{(c)}(13)$ as $\hat{P}_{i}^{(c)}=$ $R_{i}^{-1} \Phi_{i} \Delta_{i} \Psi_{i} R_{X}$. Consequently, from (13) and (3), $W_{i}$ and $A_{i}$ can be estimated by

$$
\begin{aligned}
\hat{W}_{i} & =R_{X}^{T} \Psi_{i}^{(s)} \\
\hat{A}_{i}^{(c)} & =R_{i}^{-1} \Phi_{i}^{(s)} \Delta_{i}^{(s)},
\end{aligned}
$$

with $\hat{U}_{i}=R_{X}^{-1} \Psi_{i}^{(s)} . \Delta_{i}^{(s)}$ is the diagonal matrix of the $N_{f}$ largest singular values of (17) and $\Phi_{i}^{(s)}, \Psi_{i}^{(s)}$ are the related left and right singular vectors.

Finally, from Theorem 1, enhanced signals are given by

$$
\hat{S}_{i}=X \hat{U}_{i}
$$

It is worth noting that the GEVD of the pair of (spatial) covariance matrices $\left(\hat{\Sigma}_{i}, \hat{\Sigma}_{X}\right)$ allows to estimate the spatial pattern $\hat{W}_{i}$ but also the temporal pattern $\hat{A}_{i}^{(c)}$ of ERPs since matrix $\hat{\Sigma}_{i}$ not only summarizes the spatial information about ERP, but also all temporal model information about the shape of the ERPs. This variant of $\mathrm{xDAWN}$ algorithm, which is definitively faster than the computation of two QR and one SVD used to demonstrate Theorem 1, is summarized in Algorithm 1. The choice of $N_{f_{i}}$ can be performed from the eigenvalues $\Lambda_{i}^{(s)}$ to select the signal and noise subspaces.

\section{Theoretical results and asymptotical performance}

In this section, let us assume that 
(A1) all spatio-temporal patterns $P_{i, j}$ are mono-dimensional, i.e. $\forall(i, j), P_{i, j}=\left(\mathbf{a}_{i}^{(c)}+\mathbf{a}_{i, j}^{(r)}\right) \mathbf{w}_{i}^{T}$, with $\mathbf{a}_{i}^{(c)} \in \mathbb{R}^{M_{i}}, \mathbf{a}_{i}^{(r)} \in \mathbb{R}^{M_{i}}$ and $\mathbf{w}_{i} \in \mathbb{R}^{N_{s}}$. Model (3) can then be expressed as

$$
\begin{aligned}
X & =\sum_{i=1}^{N_{e}}\left(D_{i}^{(c)} \mathbf{a}_{i}^{(c)}+D_{i}^{(r)} \mathbf{a}_{i}^{(r)}\right) \mathbf{w}_{i}^{T}+N \\
& =S W^{T}+N
\end{aligned}
$$

where $\mathbf{a}_{i}^{(r)} \in \mathbb{R}^{M_{i} K_{i}}$, with $\mathbf{a}_{i}^{(r)}=\left[\mathbf{a}_{i, 1}^{(r)^{T}}, \cdots, \mathbf{a}_{i, K_{i}}^{(r)^{T}}\right]^{T} . W=$ $\left[\mathbf{w}_{1}, \cdots, \mathbf{w}_{N_{e}}\right] \in \mathbb{R}^{N_{s} \times N_{e}}$ and $S \in \mathbb{R}^{N_{t} \times N_{e}}$ whose $i$-th column $S_{i}$ is equal to $S_{i}=\left(D_{i}^{(c)} \mathbf{a}_{i}^{(c)}+D_{i}^{(r)} \mathbf{a}_{i}^{(r)}\right)$;

(A2) the additive noise $\mathbf{n}(k)$ is a temporally white centered Gaussian vector with spatial covariance matrix $R_{n}=$ $E\left[\mathbf{n}(k) \mathbf{n}^{T}(k)\right]$ and is uncorrelated with $\mathbf{a}_{i}^{(r)} ;$

(A3) the random parts of ERP, $\mathbf{a}_{i}^{(r)}$, are centered and pairwise uncorrelated with $E\left[\mathbf{a}_{i}^{(r)} \mathbf{a}_{i}^{(r)^{T}}\right]=\delta_{i}^{2} I$, where $I$ is the identity matrix;

(A4) the common parts of ERP, $\mathbf{a}_{i}^{(c)}$, are pairwise orthogonal;

(A5) the estimated spatial filters are applied to data that haven't been used to estimate them.

Let us denote by $Y_{(-i)}$ the submatrix of $Y$ such that its $i$-th column is removed: $Y_{(-i)}=\left[Y_{:, 1}, \cdots, Y_{:, i-1}, Y_{:, i+1}, \cdots, Y_{:, N}\right]$, where $Y_{:, k}$ is the $k$-th column of $Y$. Moreover, let us define the mean signal-to-interference-plus-noise ratio (SINR) $r_{i}(\mathbf{u})$ achieved after applying the spatial filter $\mathbf{u}$ to enhance the $i$-th class of ERP by

$$
r_{i}(\mathbf{u})=\frac{\sigma(i)^{2} E\left[\left(\mathbf{u}^{T} \mathbf{w}_{i}\right)^{2}\right]}{E\left[\mathbf{u}^{T} R_{H_{(-i)}} \mathbf{u}\right]},
$$

where $R_{H_{(-i)}}$ is the covariance matrix of interferences and noise $H_{(-i)}=S_{(-i)} W_{(-i)}^{T}+N$ and $\sigma(i)^{2}=E\left[S_{i}^{T} S_{i}\right] /\left(K_{i} M_{i}\right)$

Under assumption (A1), it is well known that the optimal spatial filter (4) which maximizes the SSNR (or equivalently the SINR) of the $i$-th ERP is given by [21]

$$
\tilde{\mathbf{u}}_{i}=R_{H_{(-i)}}^{-1} \mathbf{w}_{i}
$$

The aim of this section is first to investigate the conditions such that the XDAWN framework leads to estimate the optimal spatial filter (Section 3.1) and then to study the influence on the SINR of a limited number of time samples to estimate covariance matrices used to compute the spatial filter (Section 3.2).

\subsection{Theoretical justifications: optimality of spatial filters}

In this section, we investigate the behavior of proposed xDAWN in the case of a perfect estimation of $\hat{\Sigma}_{i}$ and $\hat{\Sigma}_{X}$ defined by the expectation of covariance matrices (11) and (10)

$$
\Sigma_{i} \triangleq \frac{E\left[\hat{P}_{i}^{(c)^{T}} \hat{D}_{i}^{(c)^{T}} \hat{D}_{i}^{(c)} \hat{P}_{i}^{(c)}\right]}{N_{t}} \quad \text { and } \quad \Sigma_{X} \triangleq \frac{E\left[X^{T} X\right]}{N_{t}}
$$

From assumptions (A1)-(A4), one can express these covariance matrices as

$$
\begin{aligned}
\Sigma_{i} & =W \Gamma_{i} W^{T}+\eta_{i} R_{n}, \\
\Sigma_{X} & =W \Sigma W^{T}+R_{n},
\end{aligned}
$$

where

$$
\eta_{i}=\frac{\operatorname{Tr}\left(\hat{B}_{i} \hat{D}_{i}^{(c)^{T}} \hat{D}_{i}^{(c)} \hat{B}_{i}^{T}\right)}{N_{t}},
$$

$\Sigma$ and $\Gamma_{i}$ are two diagonal matrices whose $k$-th diagonal entries are equal to $\alpha_{k} \sigma^{2}(k)$ and $\alpha_{k} \gamma_{i}^{2}(k)$, respectively, with $\sigma(k)^{2}=$ $\sigma^{(c)}(k)^{2}+\sigma^{(r)}(k)^{2}$, where

$$
\begin{aligned}
\sigma^{(c)}(k)^{2} & =\frac{\mathbf{a}_{k}^{(c)^{T}} D_{k}^{(c)^{T}} D_{k}^{(c)} \mathbf{a}_{k}^{(c)}}{K_{k} M_{k}}, \\
\sigma^{(r)}(k)^{2} & =\frac{\operatorname{Tr}\left(D_{k}^{(c)^{T}} D_{k}^{(c)}\right)}{K_{k} M_{k}} \delta_{k}^{2}=\delta_{k}^{2}
\end{aligned}
$$

and $\gamma_{i}(k)^{2}=\gamma_{i}^{(c)}(k)^{2}+\gamma_{i}^{(r)}(k)^{2}$, where

$$
\begin{aligned}
\gamma_{i}^{(c)}(k)^{2} & =\frac{\mathbf{a}_{k}^{(c)^{T}} D_{k}^{(c)^{T}} \hat{B}_{i} \hat{D}_{i}^{(c)^{T}} \hat{D}_{i}^{(c)} \hat{B}_{i}^{T} D_{k}^{(c)} \mathbf{a}_{k}^{(c)}}{K_{k} M_{k}}, \\
\gamma_{i}^{(r)}(k)^{2} & =\frac{\operatorname{Tr}\left(D_{k}^{(r)^{T}} \hat{B}_{i} \hat{D}_{i}^{(c)^{T}} \hat{D}_{i}^{(c)} \hat{B}_{i}^{T} D_{k}^{(r)}\right)}{K_{k} M_{k}} \delta_{k}^{2} .
\end{aligned}
$$

Finally, $\alpha_{k}=\left(K_{k} M_{k}\right) / N_{t}$.

Consequently, expected spatial filter $\mathbf{u}_{i}$ provided by xDAWN algorithm maximizes the SSNR

$$
\rho_{i}(\mathbf{u})=\frac{\mathbf{u}^{T} \Sigma_{i} \mathbf{u}}{\mathbf{u}^{T} \Sigma_{X} \mathbf{u}}
$$

through the GEVD of the pair $\left(\Sigma_{i}, \Sigma_{X}\right)$, whose decomposition is given by Theorem 3 in Appendix $\mathrm{A}$.

The following theorem provides the conditions on covariance matrices $\Sigma_{i}(22)$ and $\Sigma_{X}$ (23) such that maximization of $\rho_{i}$ (u) (25) leads to the optimal spatial filter (21).

Theorem 2 (Optimal configuration). Let $\Sigma_{i}$ and $\Sigma_{X}$ be two matrices defined by (22) and (23), with $\eta_{i} \geq 0$.

The generalized eigenvector associated with the unique largest generalized eigenvalue of the pair $\left(\Sigma_{i}, \Sigma_{X}\right)$ is proportional to $R_{H_{(-i)}}^{-1} \mathbf{w}_{i}$ if and only if $\Gamma_{(-i)}=\eta_{i} \Sigma_{(-i)}$ and $\gamma_{i}(i)^{2}>$ $\eta_{i} \sigma(i)^{2}$.

See Appendix B for the proof.

The main restrictive condition of theorem 2 is $\Gamma_{(-i)}=\eta_{i} \Sigma_{(-i)}$, since it imposes that the powers of all interfering sources and the additive noise are strictly modified by the same multiplicative factor $\eta_{i}$ (24), which seems highly unlikely in practice. Indeed, it is easy to check that

\footnotetext{
${ }^{2}$ Note that, for sake of simplicity, $\Sigma_{(-i)}\left(\right.$ resp. $\left.\Gamma_{(-i)}\right)$ is the covariance matrix of $S_{(-i)}\left(\right.$ resp. $\left.\hat{D}_{i}^{(c)^{T}} \hat{B}_{i}^{T} S_{(i)}\right)$ which is thus the submatrix of $\Sigma\left(\right.$ resp. $\Gamma_{i}$ ) but its $i$-th row and column.
} 
- as soon as $\hat{D}_{k}^{(c)}$ includes the temporal samples of $D_{k}^{(c)}$ which interfere with $D_{i}^{(c)}$, then $B_{i}^{T} \hat{D}_{k}^{(c)}=0$ (with $k \neq i$ ). This means that the deterministic part of the $k$-th interfering class of ERPs does not corrupt the estimate of the $i$-th class of ERPs: $k \neq i, \Gamma_{(-i)}^{(c)}=0$;

- $\Gamma_{(-i)}^{(r)} \neq 0$ since it is the variance of the residue of interfering sources in the estimate of $i$-th spatio-temporal pattern $P_{i}^{(c)}$.

However, it is worth noting that $\eta_{i}$ and $\Gamma_{(-i)}^{(r)}$ tend towards 0 as soon as $K_{i}$ tends towards infinity, since $\hat{P}_{i}^{(c)}$ is estimated in the LMS sense. This means from theorem 2 that the xDAWN framework provides asymptotical optimal spatial filters when the number of trials $K_{i}$ tends towards infinity.

\subsection{Asymptotic performance analysis}

In this section, we investigate the influence of a limited number of time samples to estimate the covariance matrices $\Sigma_{i}$ and $\Sigma_{X}$ on the SINR.

Indeed, the estimation of matrices $\Sigma_{i}$ and $\Sigma_{X}$ from a limited number of samples leads to the matrices $\hat{\Sigma}_{i}=\Sigma_{i}+\delta \Sigma_{i}$ and $\hat{\Sigma}_{X}=\Sigma_{X}+\delta \Sigma_{X}$ (where $\delta \Sigma$. denotes the error of estimation between actual value of $\Sigma$. and its estimates $\hat{\Sigma}$. from data) leads to estimate the spatial filter $\hat{\mathbf{u}}_{i}$ which differs from $\mathbf{u}_{i}$ by $\delta \mathbf{u}_{i}$ : $\hat{\mathbf{u}}_{i}=\mathbf{u}_{i}+\delta \mathbf{u}_{i}$. Therefore, the mean SINR $r_{i}\left(\hat{\mathbf{u}}_{i}\right)$ achieved after applying the spatial filter $\hat{\mathbf{u}}_{i}$ can be expressed at the first order of perturbation as

$$
r_{i}\left(\hat{\mathbf{u}}_{i}\right)=\frac{\sigma(i)^{2}\left(\mathbf{u}_{i}^{*^{T}} \mathbf{w}_{i}\right)^{2}}{\mathbf{u}_{i}^{*^{T}} R_{H_{(-i)}} \mathbf{u}_{i}^{*}}\left(1-\delta r_{i}\right)=r_{i}\left(\mathbf{u}_{i}^{*}\right)\left(1-\delta r_{i}\right),
$$

with

$$
\begin{aligned}
\delta r_{i}=1- & \frac{\left(\mathbf{w}_{i}^{T} \mathbf{u}_{i}\right)^{2}}{\left(\mathbf{w}_{i}^{T} \mathbf{u}_{i}^{*}\right)^{2}} \frac{\mathbf{u}_{i}^{*^{T}} R_{H_{(-i)}} \mathbf{u}_{i}^{*}}{\mathbf{u}_{i}^{T} R_{H_{(-i)}} \mathbf{u}_{i}} \times \\
& \left(1-\frac{\operatorname{Tr}\left(R_{H_{(-i)}} E\left[\delta \mathbf{u}_{i} \delta \mathbf{u}_{i}^{T}\right]\right)}{\mathbf{u}_{i}^{T} R_{H_{(-i)}} \mathbf{u}_{i}}+\frac{\mathbf{w}_{i}^{T} E\left[\delta \mathbf{u}_{i} \delta \mathbf{u}_{i}^{T}\right] \mathbf{w}_{i}}{\left(\mathbf{w}_{i}^{T} \mathbf{u}_{i}\right)^{2}}\right)
\end{aligned}
$$

under the assumption that spatial filter $\hat{\mathbf{u}}_{i}$ is independent of $X$ (i.e. (A5)). It is easy to show that

$$
\begin{aligned}
E\left[\delta \mathbf{u}_{i} \delta \mathbf{u}_{i}^{T}\right] & =\Pi_{i} E\left[\delta \Sigma_{i} \mathbf{u}_{i} \mathbf{u}_{i}^{T} \delta \Sigma_{i}\right] \Pi_{i}+\Pi_{X} E\left[\delta \Sigma_{X} \mathbf{u}_{i} \mathbf{u}_{i}^{T} \delta \Sigma_{X}\right] \Pi_{X} \\
& +\Pi_{i} E\left[\delta \Sigma_{i} \mathbf{u}_{i} \mathbf{u}_{i}^{T} \delta \Sigma_{X}\right] \Pi_{X}+\Pi_{X} E\left[\delta \Sigma_{X} \mathbf{u}_{i} \mathbf{u}_{i}^{T} \delta \Sigma_{i}\right] \Pi_{i}
\end{aligned}
$$

where

$$
\begin{aligned}
\Pi_{i} & =\sum_{k=2}^{N_{e}} \frac{1}{\lambda_{1}^{(i)}-\lambda_{k}^{(i)}} \boldsymbol{\theta}_{k}^{(i)} \boldsymbol{\theta}_{k}^{(i)^{T}}+\frac{1}{\lambda_{1}^{(i)}-\eta_{i}} \Pi, \\
\Pi_{X} & =-\frac{1}{2} \mathbf{u}_{i} \mathbf{u}_{i}^{T}-\sum_{k=2}^{N_{e}} \frac{\lambda_{1}^{(i)}}{\lambda_{1}^{(i)}-\lambda_{k}^{(i)}} \boldsymbol{\theta}_{k}^{(i)} \boldsymbol{\theta}_{k}^{(i)^{T}}-\frac{\lambda_{1}^{(i)}}{\lambda_{1}^{(i)}-\eta_{i}} \Pi, \\
\Pi & =R_{n}^{-1}-R_{n}^{-1} W\left(W^{T} R_{n}^{-1} W\right)^{-1} W^{T} R_{n}^{-1},
\end{aligned}
$$

and $E\left[\delta \Sigma_{i} \mathbf{u}_{i} \mathbf{u}_{i}^{T} \delta \Sigma_{i}\right], \quad E\left[\delta \Sigma_{X} \mathbf{u}_{i} \mathbf{u}_{i}^{T} \delta \Sigma_{X}\right], \quad E\left[\delta \Sigma_{X} \mathbf{u}_{i} \mathbf{u}_{i}^{T} \delta \Sigma_{i}\right]$ and $E\left[\delta \Sigma_{i} \mathbf{u}_{i} \mathbf{u}_{i}^{T} \delta \Sigma_{X}\right]$ are expressed in Appendix C. In the same way, one can obtain the perturbations of $\hat{\mathbf{w}}_{i}=\mathbf{w}_{i}+\delta \mathbf{w}_{i}$ and $\hat{\mathbf{a}}_{i}^{(c)}=\mathbf{a}_{i}^{(c)}+\delta \mathbf{a}_{i}^{(c)}$ as

$$
\begin{gathered}
E\left[\delta \mathbf{w}_{i} \delta \mathbf{w}_{i}^{T}\right]=\Pi_{X}^{w} E\left[\delta \Sigma_{X} \mathbf{u}_{i} \mathbf{u}_{i}^{T} \delta \Sigma_{X}\right] \Pi_{X}^{w T}+\Pi_{i}^{w} E\left[\delta \Sigma_{i} \mathbf{u}_{i} \mathbf{u}_{i}^{T} \delta \Sigma_{i}\right] \Pi_{i}^{w T} \\
+\Pi_{X}^{w} E\left[\delta \Sigma_{X} \mathbf{u}_{i} \mathbf{u}_{i}^{T} \delta \Sigma_{i}\right] \Pi_{i}^{w T}+\Pi_{i}^{w} E\left[\delta \Sigma_{i} \mathbf{u}_{i} \mathbf{u}_{i}^{T} \delta \Sigma_{X}\right] \Pi_{X}^{w T}
\end{gathered}
$$

where

$$
\begin{aligned}
& \Pi_{X}^{w}=I+\Sigma_{X} \Pi_{X}, \\
& \Pi_{i}^{w}=\Sigma_{X} \Pi_{i},
\end{aligned}
$$

and

$$
\begin{aligned}
E\left[\delta \mathbf{a}_{i}^{(c)} \delta \mathbf{a}_{i}^{(c)}\right] & =\hat{B}_{i}^{T}\left(\operatorname{Tr}\left(R_{n} E\left[\delta \mathbf{u}_{i} \delta \mathbf{u}_{i}^{T}\right]\right) I+\sum_{k=1}^{N_{e}} \mathbf{w}_{k}^{T} E\left[\delta \mathbf{u}_{i} \delta \mathbf{u}_{i}^{T}\right] \mathbf{w}_{k}\right. \\
& \left.\left.\times\left(D_{k}^{(c)} \mathbf{a}_{k}^{(c)} \mathbf{a}_{k}^{(c)}{ }^{T} D_{k}^{(c)}\right)^{T}+D_{k}^{(r)} D_{k}^{(r)^{T}} \delta_{k}^{2}\right)\right) \hat{B}_{i} .
\end{aligned}
$$

It is worth noting that, since the estimations of covariance matrices are unbiased, the estimates $\hat{\mathbf{u}}_{i}, \hat{\mathbf{w}}_{i}$ and $\hat{\mathbf{a}}_{i}^{(c)}$ are also unbiased. Moreover, $E\left[\delta \mathbf{u}_{i} \delta \mathbf{u}_{i}^{T}\right]$ tends towards 0 when the number of time samples $N_{t}$ tends to infinity, which can be verified from the expression (C.1). As a consequence, under weak assumptions, the xDAWN framework leads to unbiased and consistent estimators.

\section{Relations with other methods}

In this section, we investigate the relation between the proposed $\mathrm{xDAWN}$ framework and other classical methods used to enhance ERPs.

\subsection{Principal and independent component analysis}

A classical approach used in neuroscience to enhance the ERPs is principal component analysis (PCA): it aims at estimating spatial filters such that the principal components are uncorrelated and account for as much of variance of the data as possible. As a consequence, the spatial filters are estimated from

$$
U_{i}^{(P C A)}=\arg \max _{U} \frac{\operatorname{Tr}\left(U^{T} A_{i}^{T} A_{i} U\right)}{\operatorname{Tr}\left(U^{T} U\right)},
$$

which are equal to $\mathbf{w}_{i}$ in the monodimensinal case. As pointed out in [18], the major drawback of PCA comes from the fact that it does not directly taken into account the noise $N$ and the others ERP $A_{j}(j \neq i)$. Even if the PCA enhances evoked potentials $A_{i}$, spatial filters $U_{i}^{(P C A)}$ could also largely amplify the concurrent ERPs and noise compared to $A_{i}$.

An other classical approach is independent component analysis (ICA) which aims at recovering the sources by optimizing a criterion which presents an optimum for independent sources (or at least based on an approximation of independence). For instance, SOBI $[8,22]$ estimates sources with different spectra, JADE $[8,23]$ is based on an assumption of non-Gaussian and independent sources through 4 th order cumulants or the most 
widely used algorithm in neuroscience FastICA [8, 24] which maximizes the negentropy. However, ICA and PCA algorithms are data driven methods which do not exploit the underlying ERP paradigm. Moreover, as pointed out in the introduction, these methods often fail to estimate accurately sources when their number is larger than the number of sensors (which is generally the case in EEG experiments) and they require to select (manually or automatically) the estimated components which mainly contain the ERPs.

\subsection{Common spatial pattern}

Common spatial pattern (CSP) [12, 13] aims at estimating spatial filters such that they discriminate between two classes using

$$
U_{i}^{(C S P)}=\arg \max _{U} \frac{\operatorname{Tr}\left(U^{T} \Sigma_{+} U\right)}{\operatorname{Tr}\left(U^{T}\left(\Sigma_{+}+\Sigma_{-}\right) U\right)},
$$

where $\Sigma_{+}$and $\Sigma_{-}$are the two data covariances matrices in the two conditions. Although CSP has been largely used to classify spectral data [13], it is also suitable to enhance temporal signals. Indeed, CSP is simply based on the fact that the power of the latent signal is larger in the first condition than in the second condition. Applied in the ERP context, it allows to enhance one of the classes with respect to all the others ones with

$$
\begin{aligned}
& \Sigma_{+}=\frac{1}{\left|\mathcal{I}_{i}\right|} E\left[X\left(\mathcal{I}_{i}\right)^{T} X\left(\mathcal{I}_{i}\right)\right] \\
& \Sigma_{-}=\frac{1}{\left|\mathcal{I}_{-i}\right|} E\left[X\left(\mathcal{I}_{-i}\right)^{T} X\left(\mathcal{I}_{-i}\right)\right]
\end{aligned}
$$

where $\mathcal{I}_{i}$ (resp. $\mathcal{I}_{-i}$ ) is the set of time indexes related to the $i$-th ERP (resp. others ERPs). $|\mathcal{I}|$ denotes the cardinal of set $\mathcal{I}$ and $X(\mathcal{I})$ indicates the samples of $X$ with time indices belonging in $\mathcal{I}$.

Even if the comparison between CSP and XDAWN can be unfair since CSP is not directly designed to enhance ERP, it is worth noting that both solutions of (35) and (3) are the generalized vectors associated with the largest generalized eigenvalue of $\left(\Sigma_{+}, \Sigma_{-}+\Sigma_{+}\right)$and $\left(\Sigma_{i}, \Sigma_{X}\right)$, respectively. These two methods share the same framework, i.e. generalized eigenvalue decomposition (GEVD), but differ in the pair of matrices $\left(\Sigma_{N}, \Sigma_{D}\right)$ to be analyzed: xDAWN enhances a repeated pattern time locked to stimuli while CSP is based on the increase of the signal power after stimuli which can be interpreted as a signalto-signal-plus-interference ratio (SSIR). Consequently, one can derive the same performance analysis for CSP as has been provided for $\mathrm{xDAWN}$ algorithm ${ }^{3}$.

\subsection{Canonical correlation analysis}

Finally, the xDAWN algorithm is closely related to the principal or canonical angles [20, 25, 26], which are generalizations of canonical correlation analysis (CCA) [27]. Indeed, if only one class of ERPs is assumed (even if several classes of

\footnotetext{
${ }^{3}$ Note that the detailed expressions are not given in this article due to the lack of space.
}

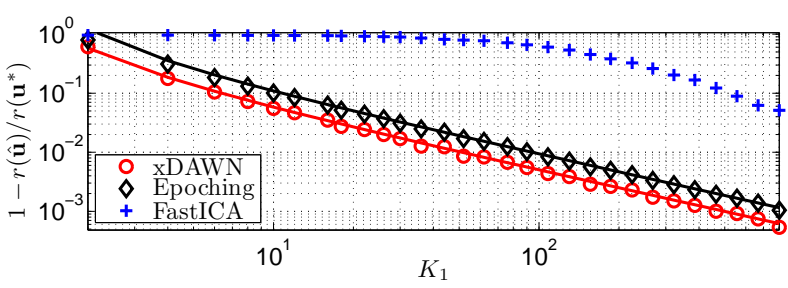

Figure 1: Asymptotical performance analysis of perturbation $\delta r$ (27) of SINR (20) with one class of stimulus $\left(N_{e}=1\right)$ and $60 \%$ of overlapping.

ERPs are involved), xDAWN algorithm, which thus sums up to classical beamforming, and CCA provide the same estimate as shown in [18]. However, as soon as several classes of ERPs are involved and assumed, the proposed xDAWN algorithm can be seen as an extension of CCA by taking into account the possible overlapping between classes of ERPs.

\section{Results}

In this section, numerical simulations are first provided to show the behavior of the xDAWN algorithm and to compare it to others classical methods (Section 5.1) before illustration on real data (Section 5.2).

\subsection{Numerical simulations}

In order to validate asymptotical performance expressions, several configurations are considered for which theoretical and numerical values are plotted. These experiments allow not only to check the correctness of the theoretical expressions and to evaluate the area of validity of the asymptotical developments but also and mainly to evaluate and to tune the parameters used in the xDAWN algorithm.

In all these analyses, the continuous lines are the theoretical asymptotic performance while markers correspond to the numerical performances obtained by averaging over 1000 independent trials. Several algorithms to estimate spatial filters are considered: xDAWN algorithm, epoching algorithm ${ }^{4}$, CSP [13] and FastICA [8, 24].

In the first set of simulations (Fig. 1), there is only one class of stimulus $\left(N_{e}=1\right)$ in the experiment. The actual duration of ERP $M_{1}$ is equal to 100 samples, spatial distribution $\mathbf{w}_{1}$ and covariance matrix $R_{n}$ are such that $\mathbf{w}_{1}^{T} R_{n}^{-1} \mathbf{w}_{1}=1$. Moreover $\alpha_{1} \sigma^{(c)}(1)^{2}=1$ and $\sigma^{(r)^{2}}=0$ so that the optimal SINR is equal to one. Finally $K_{1}$ is even such that the $(2 i-1)$-th and (2i)-th ERPs overlap by $60 \%$. It is aimed at investigating the influence of the number $K_{1}$ of trials on the estimated SINR. Obviously, the SINR increases with the number of trials. One can see that the XDAWN algorithm provides better estimates of spatial filters than epoching or FastICA, consequently xDAWN algorithm provides the best estimates of spatial filters. This

\footnotetext{
${ }^{4}$ Epoching algorithm is a variation of xDAWN algorithm where $P_{i}^{(c)}$ is estimated by averaging the signal after the stimuli onsets. This leads to replace (13) by $\hat{P}_{i}^{(c)}=D_{i}^{T} X / K_{i}$. This estimation is widely used by neuroscientists [1].
} 


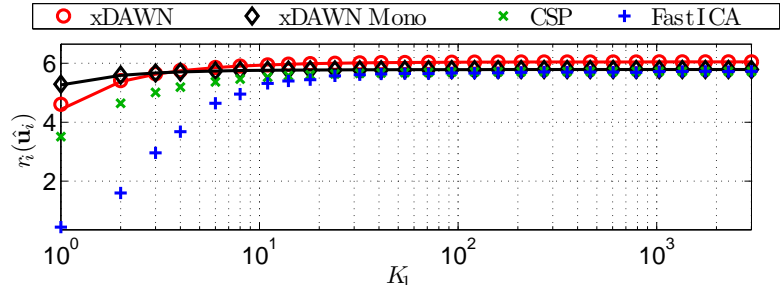

(a) Influence of $K_{1}$ on SINR (20)

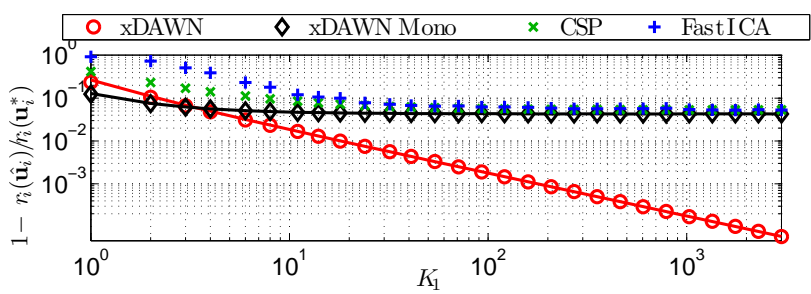

(b) Influence of $K_{1}$ on $\delta r_{i}$ (27)

Figure 2: Asymptotical performance analysis of the number of trials $K_{1}$ on SINR (20) and on perturbation $\delta r(27)$ with two classes of stimulus $\left(N_{e}=\right.$ 2 ), an overlapping between classes of $70 \%$ and within the first class of $40 \%$. Actual and estimated durations of ERPs are fixed to $100\left(M_{1}=\hat{M}_{1}=M_{2}=\right.$ $\left.\hat{M}_{2}=100\right), \mathbf{w}_{1}^{T} R_{n}^{-1} \mathbf{w}_{2}=.7$ and $\sigma^{(c)^{2}} / \sigma^{2}=.90$ for target and interfering ERPs. Optimal SINR is thus equal to 6.05 .

can be explained by the fact that the kernel $P_{1}^{(c)}$ is better estimated by (13) than by simple averaging due to the overlapping of several ERPs.

In the second set of simulations (Fig. 2), two kinds of stimuli are considered $\left(N_{e}=2\right)$. Two alternatives of xDAWN algorithm are thus involved: 'XDAWN' which assumes the true number of ERP classes and 'xDAWN Mono' for which only one class of ERP is assumed. The actual duration of ERPs $M_{1}$ and $M_{2}$ are both equal to 100 samples, spatial distributions $\mathbf{w}_{1}$ and $\mathbf{w}_{2}$ and covariance matrix $R_{n}$ are such that $\mathbf{w}_{1}^{T} R_{n}^{-1} \mathbf{w}_{1}=\mathbf{w}_{2}^{T} R_{n}^{-1} \mathbf{w}_{2}=1$ and $\mathbf{w}_{1}^{T} R_{n}^{-1} \mathbf{w}_{2}=.7$. The ratio of the numbers of target ERPs and the number of concurrent ERPs is fixed to one third $\left(K_{2}=3 K_{1}\right)$. The influence of the number $K_{1}$ of target stimuli on the SINR has been investigated. As already noticed, the xDAWN algorithm is asymptotically optimal as the number of target stimuli increases (Fig. 2(b)) while other algorithms (CSP and FastICA) provide biased estimates of the optimal spatial filters. Moreover, 'xDAWN Mono' algorithm slightly outperforms 'xDAWN' in case of few target stimuli. This can be explained by the variance of the estimate $\hat{P}_{1}^{(c)}$ with very few data.

In the third set of simulations (Fig. 3 to Fig. 5), two kinds of stimuli are considered $\left(N_{e}=2\right)$. The actual duration of ERPs $M_{1}$ and $M_{2}$ are both equal to 100 samples, spatial distributions $\mathbf{w}_{1}$ and $\mathbf{w}_{2}$ and covariance matrix $R_{n}$ are such that $\mathbf{w}_{1}^{T} R_{n}^{-1} \mathbf{w}_{1}=\mathbf{w}_{2}^{T} R_{n}^{-1} \mathbf{w}_{2}=1$. The ratio of the numbers of target ERPs and the number of concurrent ERPs is fixed to one third $\left(K_{2}=3 K_{1}\right)$. Firstly, Fig. 3 investigates the influence of temporal (Fig. 3(a)) and spatial (Fig. 3(b)) overlappings between target and concurrent stimuli. This simulation highlights that xDAWN algorithms ('xDAWN' as well as 'xDAWN Mono')

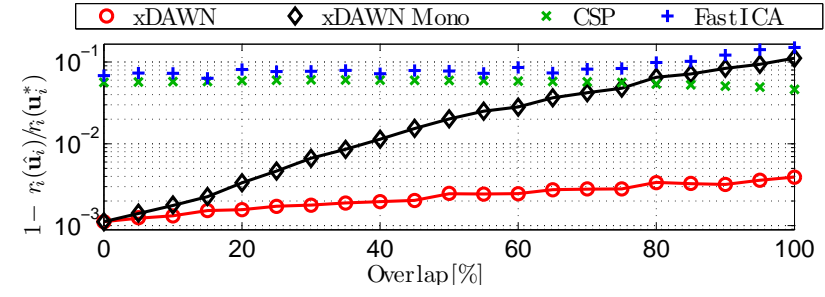

(a) Influence of overlap

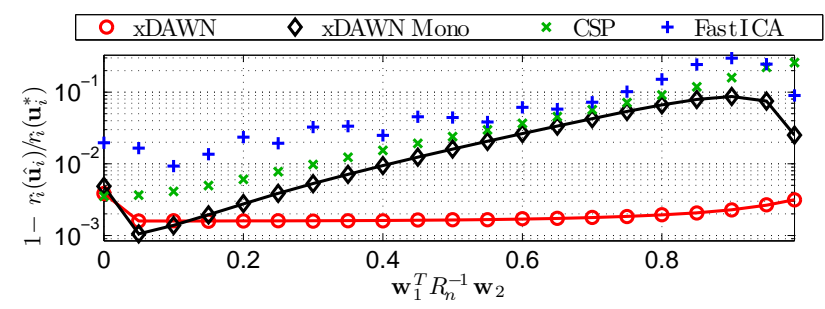

(b) Influence of $\mathbf{w}_{1}^{T} R_{n}^{-1} \mathbf{w}_{2}$

Figure 3: Asymptotical performance analysis of perturbation $\delta r$ (27) of SINR (20) with two classes of stimulus $\left(N_{e}=2\right), 100$ target stimuli $\left(K_{1}=100\right)$ so $K_{2}=300$ and $\sigma^{(c)^{2}} / \sigma^{2}=.90$ for the two classes of ERPs: influence of overlapping with $\mathbf{w}_{1}^{T} R_{n}^{-1} \mathbf{w}_{2}=.7$ (Fig. 3(a)) and of $\mathbf{w}_{1}^{T} R_{n}^{-1} \mathbf{w}_{2}$ with overlapping of $70 \%$ (Fig. 3(b)).

provide very good performance on the spatial filters estimate compared to classical CSP or FastICA, even with very confusing configurations. For instance with $100 \%$ overlapping between target and concurrent stimuli or with $\mathbf{w}_{1}^{T} R_{n}^{-1} \mathbf{w}_{2}$ close to one, 'xDAWN' provides less than $1 \%$ of performance loss compared to optimal SINR. Secondly, Fig. 4 investigates the influence of the assumed durations of ERPs $\left(\hat{M}_{1}\right.$ and $\left.\hat{M}_{2}\right)$ on the SINR. In the two simulations, xDAWN algorithm outperforms the other methods and FastICA provides quasi-constant performance since this method does not depend of this parameter. Furthermore, in Fig. 4(a) one can observe that the CSP provides the best performance when the assumed duration of ERP, $\hat{M}_{1}$, is equal to its actual value $\left(\hat{M}_{1}=100\right)$, however the CSP estimate is almost unaffected by this parameter since the performance is almost equal. Moreover, with xDAWN algorithm, the performance increases with the assumed duration of target ERPs $\left(\hat{M}_{1}\right)$ until the actual value is reached and then is constant if the assumed duration is larger than the actual $\left(\hat{M}_{1}>M_{1}=100\right)$. On the contrary, with 'xDAWN mono', the performance increases only until the estimate of $\Sigma_{1}$ is corrupted by the interfering ERPs (i.e. from $\hat{M}_{1}=1$ to 30 , since the overlapping is of $70 \%$ with $\left.M_{1}=100\right)$ and then decreases. Fig. 4(b) shows that the performance of xDAWN algorithm increases with the assumed duration of interfering ERP $\left(\hat{M}_{2}\right)$ until the part of concurrent ERPs which overlaps the target ERPs is fully included in the assumed model (i.e. $\hat{D}_{2}^{(c)}$ includes the all temporal samples of $S_{2}$ which interfere with $S_{1}$ ): $E\left[\delta r_{i}\right]$ decreases from $\hat{M}_{2}=1$ to 70 , since the overlapping between classes of ERPs is of $70 \%$ with $M_{1}=100$. Adding more temporal samples of $S_{2}$ than those which interfere with $S_{1}$ into $\hat{D}_{2}^{(c)}$ leaves the performance constant. In this simulation since 


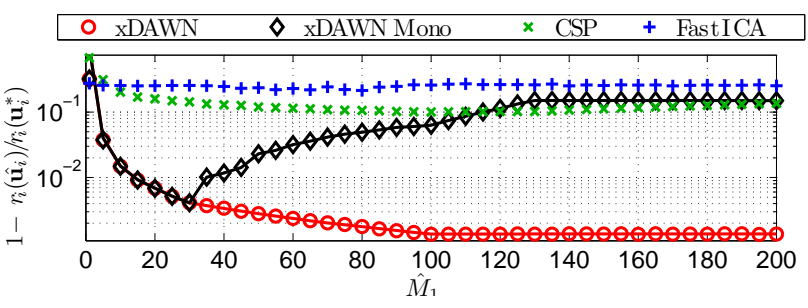

(a) Influence of $\hat{M}_{1}$

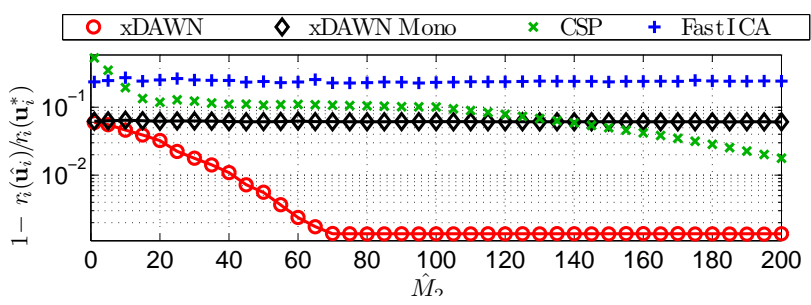

(b) Influence of $\hat{M}_{2}$

Figure 4: Asymptotical performance analysis of perturbation $\delta r$ (27) of SINR (20) with two classes of stimulus $\left(N_{e}=2\right)$ and 100 target stimuli $\left(K_{1}=100\right)$ so $K_{2}=300$ : influence of assumed duration of ERP $\hat{M}_{1}$ (Fig. 4(a)) and $\hat{M}_{2}$ (Fig. $\left.4(\mathrm{~b})\right)$ with $70 \%$ of overlap, $\mathbf{w}_{1}^{T} R_{n}^{-1} \mathbf{w}_{2}=.7$ and $\sigma^{(c)^{2}} / \sigma^{2}=.90$ for target and interfering ERPs.

xDAWN mono is independent of $\hat{M}_{2}$, its performance is obviously equal. These two simulations show that the xDAWN algorithm provides a good behavior with respect to the assumed durations of ERPs: in practice, it is even better to overestimate them since the performance is then the same as the performance achieved by the actual values. Finally, in Fig. 5 we can investigate the influence of the stochastic temporal patterns in ERPs by varying the ratio $\sigma^{(c)^{2}}(i) / \sigma(i)^{2}$ for both classes of ERPs: the global power of ERPs is remained constant, only the repartition of the power between common and stochastic pattern varies. In these two simulations, CSP and FastICA provide quite constant performance: indeed, these two methods do not assume anything about temporal patterns but the fact that the performance is slightly better with a low common temporal pattern (i.e. $\sigma^{(c)^{2}}(i) / \sigma(i)^{2}$ close to 0 ) is simply due to a better estimation of statistics since samples are thus independent which is not the case when $\sigma^{(c)^{2}}(i) / \sigma(i)^{2}$ is close to 1. In Fig. 5(a), since $\hat{M}_{2}=M_{2}$ thus $\hat{B}_{1}^{T} D_{2}=0$ with xDAWN algorithm; as a consequence, $x D A W N$ algorithm is independent of $\sigma^{(c)^{2}}(2) / \sigma(2)^{2}$, leading thus to constant performance. On the contrary, the performance of XDAWN mono, which assumes that there are no interfering ERPs, decreases with the increase of the power of the common ERPs. In this simulation, considering the case of $\sigma^{(c)^{2}}(2) / \sigma(2)^{2}$ close to 0 is very interesting since this configuration corresponds to the case of a single class of ERPs $\left(N_{e}=1\right)$. In this specific case, 'xDAWN mono' slightly outperforms xDAWN: indeed, xDAWN (resp. 'xDAWN mono') assumes that there are two (resp. one) classes of ERPs while its actual value is one. This simulation shows that $\mathrm{XDAWN}$ is quite robust to an error of modeling due to an overestimation of the actual number of classes of ERPs. Moreover, Fig. 5(b)

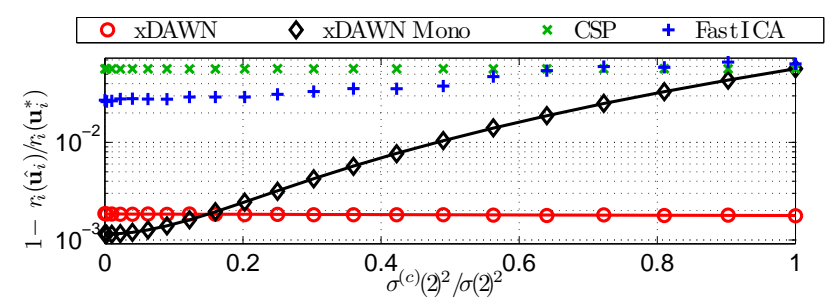

(a) Influence of $\mathbf{a}_{2}^{(r)}$

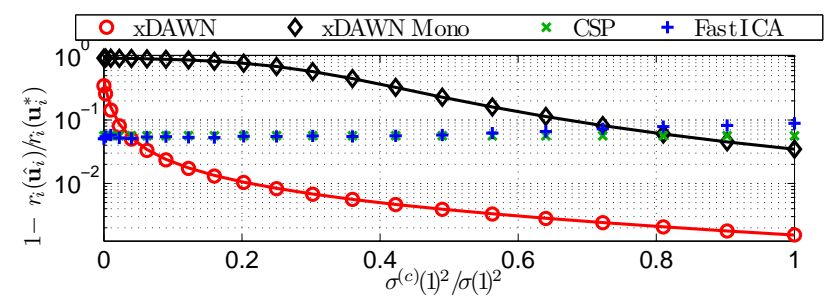

(b) Influence of $\mathbf{a}_{1}^{(r)}$

Figure 5: Asymptotical performance analysis of perturbation $\delta r$ (27) of SINR (20).

highlights that the performance of xDAWN obviously increases with the ratio $\sigma^{(c)^{2}}(1) / \sigma(1)^{2}$ (i.e. when the power of the common target pattern increases) since it is the main assumption of xDAWN framework.

\subsection{Application to real data}

In this section, the behavior of xDAWN algorithm is illustrated on actual electro-encephalograhic (EEG) data. EEG data has been recorded from 29 scalp electrodes placed at standard positions of an extended $10 * 20$ international system referenced to the nose and grounded to the forehead. All impedances have been kept below 10kOhms. Signals have been sampled at $500 \mathrm{~Hz}$ using a BrainAmp amplifier (BrainProducts $\mathrm{GmBH}$, Munich). The EEG data has been collected using the BCI2000 system with the P300 speller scenario [28]. A P300 speller is a kind of brain computer interface (BCI) which allows the user to spell sequentially symbols without any muscular control [29]. The user looks at a screen on which a $6 \times 6$ matrix regroups the 36 symbols. The rows and columns are randomly intensified and to select a symbol, the user has to focus his/her attention on the symbol he/she wants to spell. When the row or the column corresponding to the target symbol, the user's brain elicits a P300 ERP. The raw signals have been band-passed filtered with a fourth order Butterworth bandpass filter whose cutoff frequencies are $1 \mathrm{~Hz}$ and $20 \mathrm{~Hz}$.

Figure 6 displays the estimated temporal common pattern $\hat{A}_{i}^{(c)}$ related to the P300 ERP by the xDAWN algorithm and its 'xDAWN Mono' variant. As one can see, the proposed method ('xDAWN') slightly improves the estimate of the target common pattern $A_{i}^{(c)}$ : with the 'xDAWN Mono method, there exist oscillations at the same frequency as the rows/columns intensifications $(5.6 \mathrm{~Hz})$ of the P300 speller. Indeed, the temporal pattern related to target stimuli is superimposed on with a quasiperiodic signal at the same frequency as the intensifications of 


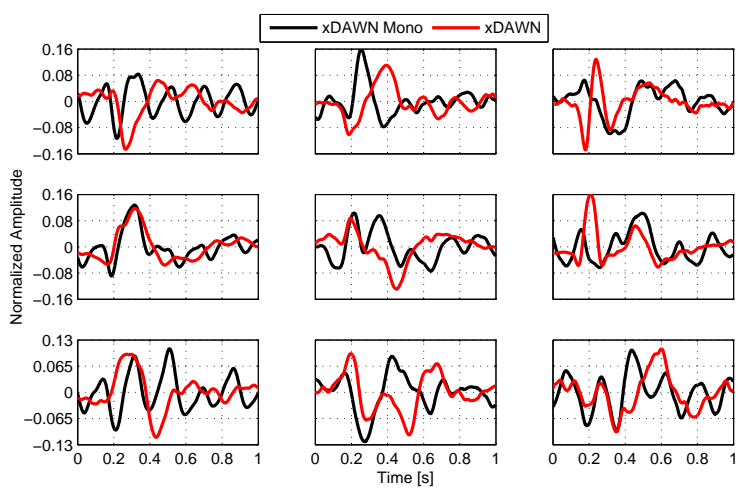

Figure 6: Temporal common pattern $A_{1}^{(c)}$ for the three subjects. Each row corresponds to one subject, and each column corresponds to one of the three dimensions of $A_{1}^{(c)}$ (the $i$ th column correspond to the $i$ th dimension).

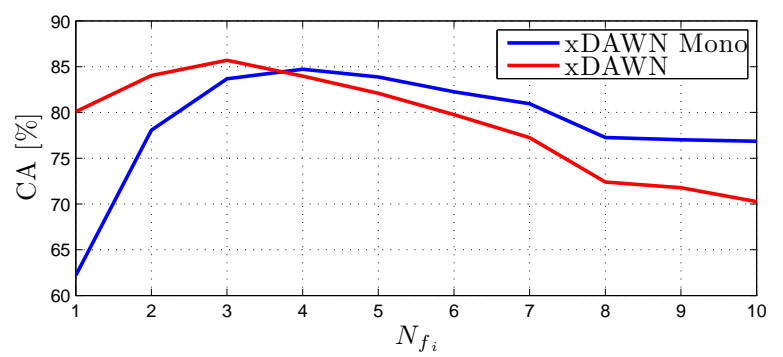

Figure 7: Classification accuracy (CA) of the P300 speller versus the number of spatial filters $\left(N_{f_{i}}\right)$.

the rows/columns of the P300 speller BCI. On the contrary, with the 'xDAWN' method, these undulations are drastically reduced. This improvement of the estimates of the common temporal pattern is also observed on the estimates of the spatial filters. Indeed, Figure 7 reports the average classification accuracy $(\mathrm{CA})$ of the $\mathrm{P} 300 \mathrm{BCI}$ prediction for the three subjects with respect to the number of spatial filters. It is worth noting that with only one spatial filter, 'xDAWN' algorithm outperforms 'xDAWN Mono', highlighting the importance of modeling the P300 ERP as well as the interfering ERP related to all stimulation flashes. The CA shows that the P300 ERPs lie in a small dimensional space since the $\mathrm{CA}$ is optimal with three spatial filters.

Figures 8 and 9 show the influence of the assumed duration of ERP $\hat{M}_{1}$ and $\hat{M}_{2}$, respectively, on the estimate $\hat{A}_{1}^{(c)}$ related to the P300 ERP. The estimates $\hat{A}_{1}^{(c)}$ are robust to the assumed duration $\hat{M}_{1}$ (Fig. 8), since the estimates differ only slightly against $\hat{M}_{1}$. Moreover, the estimates have converged for $\hat{M}_{1} \geq 400$ : increasing $\hat{M}_{1}$ does not modify the estimates. The xDAWN alogrithm is also robust to an overestimation of the assumed duration $\hat{M}_{2}$ (Fig. 9): in this experiment choosing $\hat{M}_{2}$ larger than 90 (i.e. the duration of the interstimulus interval) does not change the shape of the estimated $\hat{A}_{1}^{(c)}$. These two experiments confirm the theoretical experiments (Fig. 4) that it is better to overestimate the duration of the assumed ERPs than to underestimate them.
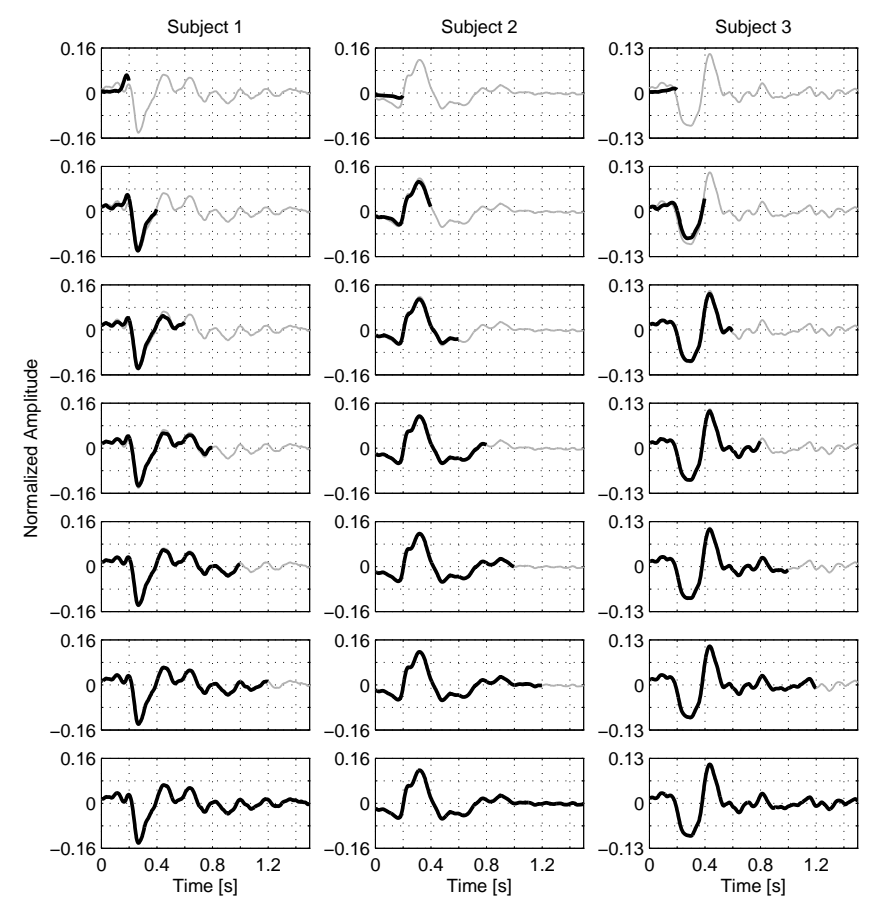

Figure 8: Influence of the assumed duration of ERP $\hat{M}_{1}$, with $\hat{M}_{2}=500$ (i.e. 1s). Each column is related to one subject and from top to bottom $\hat{M}_{1} \in\{100,200,300,400,500,600,750\}$ (i.e. .2, .4, .6, .8, 1 and 1.5 seconds). The estimated temporal common pattern $\hat{A}_{1}^{(c)}$ is the black continuous line, the light gray line is the estimate for $\hat{M}_{1}=750$.
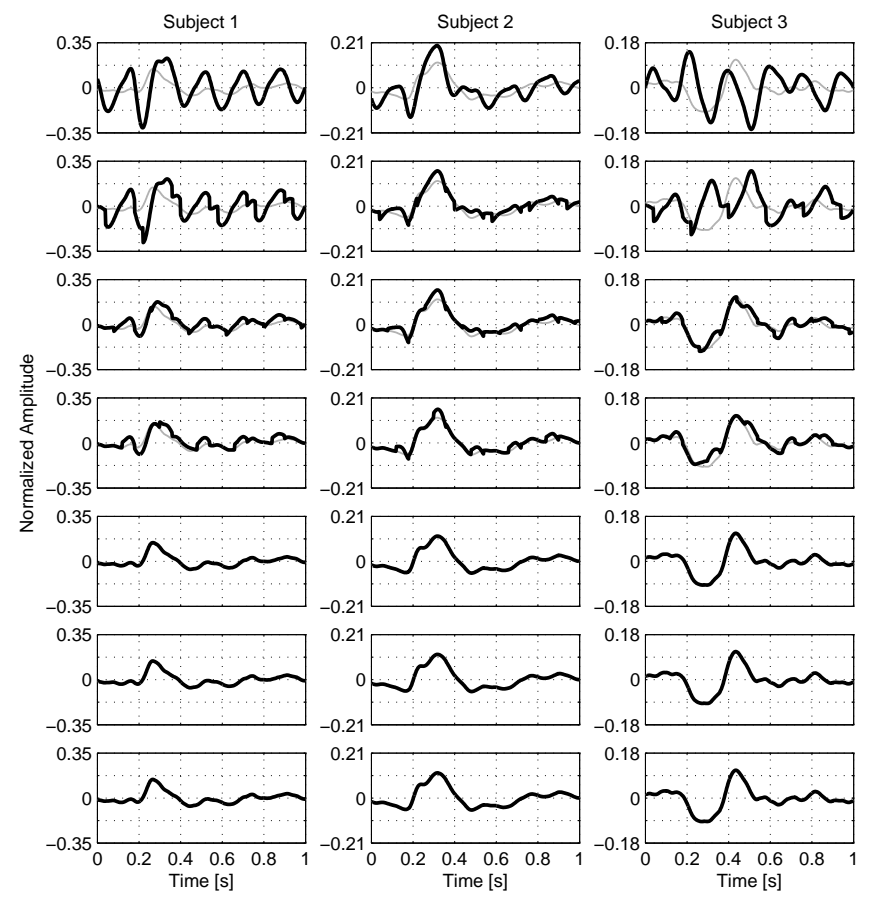

Figure 9: Influence of the assumed duration of ERP $\hat{M}_{2}$, with $\hat{M}_{1}=500$ (i.e. 1s). Each column is related to one subject and from top to bottom $\hat{M}_{2} \in\{0,20,40,60,90,120,500\}$ (i.e. $0, .04, .08, .12, .18, .24,1 \mathrm{sec}-$ onds). The estimated temporal common pattern $\hat{A}_{1}^{(c)}$ is the black continuous line, the light gray line is the estimate for $\hat{M}_{2}=.24$. 


\section{Conclusions and perspectives}

In this article, theoretical spatial filters and asymptotical performance analysis of the proposed xDAWN framework are provided. The proposed $x D A W N$ framework estimates a factorization of the space spanned by a repeated spatio-temporal pattern time-locked to target stimuli. The formulation of the decomposition is given in a closed form through a generalized eigenvalue decomposition of a pair of particular covariance matrices which only requires the knowledge of stimuli onsets. It provides the full factorization composed of the temporal patterns and its spatial distribution over sensors as well as the related spatial filters which leads to the maximum SINR. A theoretical analysis of the xDAWN framework shows that under weak assumptions the xDAWN algorithm is asymptotically optimal to estimate the spatial filters and decomposition of spatio-temporal patterns. Moreover, the finite sample effect is calculated theoretically in a closed form and validated by simulations: the xDAWN framework leads to an unbiased and consistent estimator of optimal spatial filters. These results allow firstly to demonstrate the good behavior of the proposed xDAWN algorithm compared to CSP and FastICA even with complex configurations if model (3) is satisfied. The theoretical analysis of xDAWN algorithm has shown that it has the property to be robust with respect to model parameter values. In addition, these results are useful to tune parameters of the experiments (for instance, the number of target stimulus repetitions needed to obtain a desired SINR). Finally, illustrations on real EEG data show that xDAWN algorithm outperforms classical spatial filtering methods such as CSP or FastICA in a P300 speller BCI context.

Future works will deal with the automatic estimation of model parameters (for instance time duration of ERPs). Moreover, the latency of each single ERP can slightly vary over the experiment as well as its amplitude: future works will also embedded their estimations into the framework.

\section{Appendix A. Pair beamformer defined by generalized} eigenvalue decomposition of $\left(\Sigma_{i}, \Sigma_{X}\right)$

Theorem 3 (Pair beamformer defined by GEVD of $\left(\Sigma_{i}, \Sigma_{X}\right)$ ). Let consider $\left(\Sigma_{i}, \Sigma_{X}\right)$ defined by (22) and (23), where $\Sigma_{X}$ and $R_{n}$ are definite positive matrices, such that $\Sigma_{i} \neq \mu \Sigma_{X}$.

Moreover let assume that

$$
\Gamma_{i}=\left(\begin{array}{cc}
\Gamma_{i}^{\prime} & 0 \\
0 & \Gamma_{i}^{\prime \prime}
\end{array}\right) \quad \text { and } \quad \Sigma=\left(\begin{array}{cc}
\Sigma^{\prime} & 0 \\
0 & \Sigma^{\prime \prime}
\end{array}\right)
$$

are two blocks decompositions ${ }^{5}$ of matrices $\Gamma_{i}$ and $\Sigma$ such that $\Gamma_{i}^{\prime} \neq \eta_{i} \Sigma^{\prime}$ and $\Gamma_{i}^{\prime \prime}=\eta_{i} \Sigma^{\prime \prime}$, with $\Gamma_{i}^{\prime} \in \mathbb{R}^{N_{r} \times N_{r}}$. Finaly, let decompose $W$ such that $W=\left[W^{\prime}, W^{\prime \prime}\right]$, with $W^{\prime} \in \mathbb{R}^{N_{s} \times N_{r}}$.

The GEVD of the pair $\left(\Sigma_{i}, \Sigma_{X}\right)$ has the following decomposition

- $\eta_{i}$ for generalized eigenvalue $\left(N_{s}-N_{r}\right)$ times whose associated generalized eigenvectors are orthogonal to $\mathbf{w}_{i}$, $\forall i \in\left\{1, \cdots, N_{r}\right\}$

\footnotetext{
${ }^{5}$ Without lose of generality, it is assumed that the entries of $\Gamma_{i}$ such that $\Gamma_{i}^{\prime} \neq \eta_{i} \Sigma^{\prime}$ are the $N_{r}$ first ones.
}

- $N_{r}$ generalized eigenvalues $\lambda_{k} \neq \eta_{i}$ whose associated generalized eigenvectors are expressed as linear combinaisons of $R_{n}^{-1} \mathbf{w}_{j}$ :

$$
\mathbf{v}_{k}=R_{n}^{-1} W \boldsymbol{\beta}_{k}
$$

with $\boldsymbol{\beta}_{k}=\left[\beta_{k, 1}, \cdots, \beta_{k, N_{e}}\right]^{T}=\left[\boldsymbol{\beta}_{k}^{\prime^{T}}, \boldsymbol{\beta}_{k}^{\prime{ }^{T}}\right]^{T}$. $\boldsymbol{\beta}_{k}^{\prime}$ is thus nontrivial solutions of

$$
\Sigma_{i}^{w} \beta_{k}^{\prime}=\lambda_{k} \Sigma_{X}^{w} \beta_{k}^{\prime},
$$

with $\Sigma_{i}^{w}=W^{\prime T} R_{H}^{-T} \Sigma_{i} R_{H}^{-1} W^{\prime}, \Sigma_{X}^{w}=W^{\prime T} R_{H}^{-T} \Sigma_{X} R_{H}^{-1} W^{\prime}$ and $\beta_{k}^{\prime \prime}=-\Sigma^{\prime \prime} W^{\prime \prime} R_{H}^{-1} W^{\prime} \beta_{k}^{\prime}$, where $R_{H}=R_{n}+W^{\prime \prime} \Sigma^{\prime \prime} W^{\prime \prime}{ }^{T}$.

Proof The GEVD of the pair $\left(\Sigma_{i}, \Sigma_{X}\right)$,

$$
\forall k \in\left\{1, \cdots, N_{s}\right\}, \quad \Sigma_{i} \mathbf{v}_{k}=\lambda_{k} \Sigma_{X} \mathbf{v}_{k},
$$

is equivalent to the GEVD of

$$
W^{\prime}\left(\Gamma_{i}^{\prime}-\lambda_{k} \Sigma^{\prime}\right){W^{\prime}}^{T} \mathbf{v}_{k}=\left(\lambda_{k}-\eta_{i}\right)\left(W^{\prime \prime} \Sigma^{\prime \prime} W^{\prime{ }^{T}}+R_{n}\right) \mathbf{v}_{k} .
$$

From this latter expression, it is easy to show that

- $\lambda_{k}=\eta_{i}, N_{s}-N_{r}$ times and related $\mathbf{v}_{k}$ are all orthogonal to $\mathbf{w}_{i}, \forall i \in\left\{1, \cdots, N_{e}\right\}$;

- $\lambda_{k} \neq \eta_{i}, N_{r}$ times and related $\mathbf{v}_{k}$ are linear combinaison of $R_{n}^{-1} \mathbf{w}_{i}$, using the left multiplication of (A.2) by $\frac{1}{\lambda_{k}-\eta_{i}} R_{n}^{-1}$.

Moreover, left multiplication of (A.2) by $\left(W^{\prime \prime} \Sigma^{\prime \prime} W^{\prime \prime}+R_{n}\right)^{-1}$ and Woodbory identities [30] lead to write

$$
\begin{aligned}
\mathbf{v}_{k} & =\left(W^{\prime \prime} \Sigma^{\prime \prime} W^{\prime \prime}+R_{n}\right)^{-1} W^{\prime} \boldsymbol{\beta}_{k}^{\prime} \\
& =R_{n}^{-1} W^{\prime} \boldsymbol{\beta}_{k}^{\prime}-R_{n}^{-1} W^{\prime \prime} \Sigma^{\prime \prime} W^{\prime \prime}\left(W^{\prime \prime} \Sigma^{\prime \prime} W^{\prime \prime}+R_{n}\right)^{-1} W^{\prime} \boldsymbol{\beta}_{k}^{\prime} \\
& =R_{n}^{-1} W \boldsymbol{\beta}_{k},
\end{aligned}
$$

with $\boldsymbol{\beta}_{k}=\left[\boldsymbol{\beta}_{k}^{\prime^{T}}, \boldsymbol{\beta}_{k}^{\prime^{T}}\right]^{T}$.

Finally by injecting $\mathbf{v}_{k}=\left(W^{\prime \prime} \Sigma^{\prime \prime} W^{\prime \prime}{ }^{T}+R_{n}\right)^{-1} W^{\prime} \boldsymbol{\beta}_{k}^{\prime}$ in $\Sigma_{i} \mathbf{v}_{k}=$ $\lambda_{k} \Sigma_{X} \mathbf{v}_{k}$, one can expressed $\boldsymbol{\beta}_{k}^{\prime}$ as the non-trivial solutions of (A.1).

\section{Appendix B. Proof of theorem 2}

Proof Let $\Sigma_{i}$ and $\Sigma_{X}$ be two matrices of $\mathbb{R}^{N_{s} \times N_{s}}$ defined by (22) and (23). Theorem 3 shows that this pair of matrices has only two distinct generalized eigenvalues: $\eta_{i}$ degenerated $N_{s}-1$ times and $\lambda_{k} \neq \eta_{i}$ once.

Let us suppose that $\eta_{i} \geq 0, \Gamma_{(-i)}=\eta_{i} \Sigma_{(-i)}$ and $\gamma_{i}(i)^{2}>$ $\eta_{i} \sigma(i)^{2}$. Let denote $\mathbf{v}_{k}$ and $\lambda_{k}$ the generalized eigenvectors and eigenvalues of the pair $\left(\Sigma_{i}, \Sigma_{X}\right): \forall k, \Sigma_{i} \mathbf{v}_{k}=\lambda_{k} \Sigma_{X} \mathbf{v}_{k}$.

In this case,

$$
\begin{aligned}
\Sigma_{i} \mathbf{v}_{k} & =\alpha_{i} \gamma_{i}(i)^{2}\left(\mathbf{w}_{i}^{T} \mathbf{v}_{k}\right) \mathbf{w}_{i}+\eta_{i} R_{H_{(-i)}} \mathbf{v}_{k}, \\
\Sigma_{X} \mathbf{v}_{k} & =\alpha_{i} \sigma(i)^{2}\left(\mathbf{w}_{i}^{T} \mathbf{v}_{k}\right) \mathbf{w}_{i}+R_{H_{(-i)}} \mathbf{v}_{k},
\end{aligned}
$$


where $R_{H_{(-i)}}=W_{(-i)} \Sigma_{(-i)} W_{(-i)}^{T}+R_{n}$.

Theorem 3 shows that the eigenvector associated with the unique generalized eigenvalue different from $\eta_{i}$ can be expressed as $\mathbf{v}_{k} \propto R_{H_{(-i)}}^{-1} \mathbf{w}_{i}$. In this case, the associated generalized eigenvalue satisfies

$$
\begin{aligned}
\lambda_{k} & =\frac{\mathbf{v}_{k}^{T} \Sigma_{i} \mathbf{v}_{k}}{\mathbf{v}_{k}^{T} \Sigma_{X} \mathbf{v}_{k}} \\
& =\frac{\eta_{i}+\alpha_{i} \gamma_{i}(i)^{2} \mathbf{w}_{i}^{T} R_{H_{(-i)}}^{-1} \mathbf{w}_{i}}{1+\alpha_{i} \sigma(i)^{2} \mathbf{w}_{i}^{T} R_{H_{(-i)}}^{-1} \mathbf{w}_{i}} \\
& >\eta_{i}
\end{aligned}
$$

since $\eta_{i} \sigma(i)^{2}<\gamma_{i}(i)^{2}$.

This demonstrate that the generalized eigenvector associated with the unique largest generalized eigenvalue of the pair $\left(\Sigma_{i}, \Sigma_{X}\right)$ is proportional to $R_{H_{(-i)}^{-1}}^{-1} \mathbf{w}_{i}$.

$\Rightarrow$ Let us suppose that the generalized eigenvector $\mathbf{v}_{k}$ associated with the unique largest eigenvalue of the pair $\left(\Sigma_{i}, \Sigma_{X}\right)$ is proportional to $R_{H_{(-i)}}^{-1} \mathbf{w}_{i}$. Using Woodbury identities [20], one can note that

$$
\begin{aligned}
\Sigma_{i} \mathbf{v}_{k}=\left(\alpha_{i} \gamma_{i}(i)^{2}\left(\mathbf{w}_{i}^{T} R_{H_{(-i)}}^{-1} \mathbf{w}_{i}\right)+\eta_{i}\right) \mathbf{w}_{i} & \\
& +W_{(-i)}\left(\Gamma_{(-i)}-\eta_{i} \Sigma_{(-i)}\right) W_{(-i)}^{T} R_{H_{(-i)}}^{-1} \mathbf{w}_{i}
\end{aligned}
$$

and

$$
\Sigma_{X} \mathbf{v}_{k}=\left(1+\alpha_{i} \sigma(i)^{2} \mathbf{w}_{i}^{T} R_{H_{(-i)}}^{-1} \mathbf{w}_{i}\right) \mathbf{w}_{i}
$$

Since $\Sigma_{X} \mathbf{v}_{k}$ lies in the span of $\mathbf{w}_{i}$ and since $R_{H_{(-i)}}$ is a full rank matrix, this implies that $\Gamma_{(-i)}=\eta_{i} \Sigma_{(-i)}$. Moreover, due to the uniqueness of the largest generalized eigenvalue $\lambda_{k}$, one can write that

$$
\begin{aligned}
\lambda_{k} & =\frac{\mathbf{v}_{k}^{T} \Sigma_{i} \mathbf{v}_{k}}{\mathbf{v}_{k}^{T} \Sigma_{X} \mathbf{v}_{k}} \\
& =\frac{\eta_{i}+\alpha_{i} \gamma_{i}(i)^{2} \mathbf{w}_{i}^{T} R_{H}^{-1} \mathbf{w}_{i}}{1+\alpha_{i} \sigma(i)^{2} \mathbf{w}_{i}^{T} R_{H}^{-1} \mathbf{w}_{i}} .
\end{aligned}
$$

As a consequence, $\lambda_{k}>\eta_{i}$ implies that $\gamma_{i}(i)^{2}>\eta_{i} \sigma(i)^{2}$, finishing the demonstration.

\section{Appendix C. Expressions of $\boldsymbol{E}\left[\delta \boldsymbol{\Sigma}_{\alpha} \mathbf{u}_{i} \mathbf{u}_{i}^{T} \delta \boldsymbol{\Sigma}_{\beta}\right]$}

After some computation, one can obtain that

$$
\begin{gathered}
E\left[\delta \Sigma_{\alpha} \mathbf{u}_{i} \mathbf{u}_{i}^{T} \delta \Sigma_{\beta}\right]=\frac{1}{N_{t}}\left(\left(\mathbf{u}_{i}^{T} R_{n} \mathbf{u}_{i}\right) \Sigma_{\alpha, \beta}+R_{n} \mathbf{u}_{i} \mathbf{u}_{i}^{T} \Sigma_{\alpha, \beta}\right. \\
\left.+\left(\mathbf{u}_{i}^{T}\left(\Sigma_{\alpha, \beta}-\eta_{\alpha, \beta} R_{n}\right) \mathbf{u}_{i}\right) R_{n}+\left(\Sigma_{\alpha, \beta}-\eta_{\alpha, \beta} R_{n}\right) \mathbf{u}_{i} \mathbf{u}_{i}^{T} R_{n}\right) \\
+W E\left[\delta \Sigma_{S^{(r)}}^{(\alpha)} W^{T} \mathbf{u}_{i} \mathbf{u}_{i}^{T} W \delta \Sigma_{S^{(r)}}^{(\beta)}\right] W^{T} \\
+\frac{1}{N_{t}^{2}} W\left(E\left[S^{(r)^{T}} F_{\alpha} S^{(c)} W^{T} \mathbf{u}_{i} \mathbf{u}_{i}^{T} W S^{(r)^{T}} F_{\beta} S^{(c)}\right]\right. \\
+E\left[S^{(c)^{T}} F_{\alpha} S^{(r)} W^{T} \mathbf{u}_{i} \mathbf{u}_{i}^{T} W S^{(c)^{T}} F_{\beta} S^{(r)}\right] \\
+E\left[S^{(r)^{T}} F_{\alpha} S^{(c)} W^{T} \mathbf{u}_{i} \mathbf{u}_{i}^{T} W S^{(c)^{T}} F_{\beta} S^{(r)}\right] \\
\left.+E\left[S^{(c)^{T}} F_{\alpha} S^{(r)} W^{T} \mathbf{u}_{i} \mathbf{u}_{i}^{T} W S^{(r)^{T}} F_{\beta} S^{(c)}\right]\right) W^{T},
\end{gathered}
$$

where $(\alpha, \beta) \in\{X, i\}^{2}$ and

- $F_{X}$ is the identity matrix and $F_{i}=\hat{B}_{i} \hat{D}_{i}^{(c)^{T}} \hat{D}_{i}^{(c)} \hat{B}_{i}^{T}$;

- $\Sigma_{X, X}=\Sigma_{X}, \Sigma_{i, X}=\Sigma_{X, i}=\Sigma_{i}$ and $\Sigma_{i, i}=W \Gamma_{i}^{\prime} W^{T}+\eta_{i}^{\prime} R_{n}$ with

$$
\begin{aligned}
\eta_{i}^{\prime} & =\frac{\operatorname{Tr}\left(F_{i}^{2}\right)}{N_{t}}, \\
\Gamma_{i}^{\prime} & =\operatorname{diag}\left(\frac{\mathbf{a}_{k}^{(c)^{T}} D_{k}^{(c)^{T}} F_{i}^{2} D_{k}^{(c)} \mathbf{a}_{k}^{(c)}}{N_{t}}+\frac{\operatorname{Tr}\left(D_{k}^{(r)^{T}} F_{i}^{2} D_{k}^{(r)}\right)}{N_{t}} \delta_{k}^{2}\right) ;
\end{aligned}
$$

- $\eta_{X, X}=1, \eta_{i, X}=\eta_{X, i}=\eta_{i}$ and $\eta_{i, i}=\eta_{i}^{\prime}$.

Moreover, let us defined $\mathbf{v}=W^{T} \mathbf{u}$,

$$
\begin{aligned}
E\left[S^{(c)^{T}} F_{\alpha} S^{(r)} \mathbf{v v}^{T} S^{(r)^{T}} F_{\beta} S^{(c)}\right] & =\sum_{k=1}^{N_{e}} \delta_{k}^{2} v_{k}^{2} S^{(c)^{T}} F_{\alpha} D_{k}^{(r)} D_{k}^{(r)^{T}} F_{\beta} S^{(c)}, \\
E\left[S^{(r)^{T}} F_{\alpha} S^{(c)} \mathbf{v v}^{T} S^{(c)^{T}} F_{\beta} S^{(r)}\right] & =\operatorname{diag}\left(\delta_{k}^{2} \mathbf{v}^{T} S^{(c)^{T}} F_{\alpha} D_{k}^{(r)} D_{k}^{(r)^{T}} F_{\beta} S^{(c)} \mathbf{v}\right), \\
E\left[S^{(r)^{T}} F_{\alpha} S^{(c)} \mathbf{v v}^{T} S^{(r)^{T}} F_{\beta} S^{(c)}\right] & =\left[\begin{array}{c}
\delta_{1}^{2} \mathbf{v}^{T} S^{(c)^{T}} F_{\alpha} D_{1}^{(r)} D_{1}^{(r)^{T}} F_{\beta} S^{(c)} v_{1} \\
\vdots \\
\vdots \\
\delta_{N_{e}}^{2} \mathbf{v}^{T} S^{(c)^{T}} F_{\alpha} D_{N_{e}}^{(r)} D_{N_{e}}^{(r)} F_{\beta} S^{(c)} v_{N_{e}}
\end{array}\right] \\
E\left[S^{(c)^{T}} F_{\alpha} S^{(r)} \mathbf{v}^{T} S^{(c)^{T}} F_{\beta} S^{(r)}\right] & =E\left[S^{(c)^{T}} F_{\alpha} S^{(r)} \mathbf{v}^{T} S^{(r) T^{T}} F_{\beta} S^{(c)}\right]^{T} .
\end{aligned}
$$

Finally, for all $i \neq j$

$$
E\left[\delta \Sigma_{S^{(r)}}^{(\alpha)} W^{T} \mathbf{u}_{i} \mathbf{u}_{i}^{T} W \delta \Sigma_{S^{(r)}}^{(\beta)}\right]_{i, j}=\frac{\operatorname{Tr}\left(D_{i}^{(r)^{T}} F_{\alpha} D_{j}^{(r)} D_{j}^{(r)} F_{\beta}^{T} D_{i}^{(r)}\right)}{N_{t}^{2}} \delta_{i}^{2} \delta_{j}^{2} v_{i} v_{j},
$$

and

$$
\begin{gathered}
E\left[\delta \Sigma_{S^{(r)}}^{(\alpha)} W^{T} \mathbf{u}_{i} \mathbf{u}_{i}^{T} W \delta \Sigma_{S^{(r)}}^{(\beta)}\right]_{i, i}=\frac{\operatorname{Tr}\left(D_{i}^{(r)^{T}} F_{\alpha} D_{i}^{(r)} D_{i}^{(r)^{T}} F_{\beta} D_{i}^{(r)}\right)}{N_{t}^{2}} \delta_{i}^{4} v_{i}^{2} \\
+\sum_{k=1}^{N_{e}} \frac{\operatorname{Tr}\left[D_{i}^{(r)^{T}} F_{\alpha} D_{k}^{(r)} D_{k}^{(r)^{T}} F_{\beta} D_{i}^{(r)}\right]}{N_{t}^{2}} \delta_{i}^{2} \delta_{k}^{2} v_{k}^{2} .
\end{gathered}
$$

\section{References}

[1] E. Niedermeyer, F. H. Lopes da Silva, Electroencephalography: Basic principles, clinical applications, and related fields, Lippincott Williams \& Wilkins, 2005.

[2] S. J. Luck, An Introduction to the Event-Related Potential Technique, The MIT Press, 2005.

[3] M. Hoke, B. Ross, R. Wickesberg, B. Lütkenhöner, Weighted averaging theory and application to electric response audiometry, Electroen. Clin. Neuro. 57 (5) (1984) 484-489.

[4] C. Jutten, J. Hérault, Blind separation of sources. Part I: An adaptive algorithm based on a neuromimetic architecture, Signal Processing 24 (1) (1991) $1-10$.

[5] P. Comon, Independent component analysis, a new concept?, Signal Processing 36 (3) (1994) 287-314.

[6] J.-F. Cardoso, Blind signal separation: statistical principles, Proceedings of the IEEE 86 (10) (1998) 2009-2025.

[7] A. Hyvärinen, Blind Source Separation by Nonstationarity of Variance: A Cumulant-Based Approach, IEEE Trans. Neural Netw. 12 (6) (2001) 1471-1474.

[8] P. Comon, C. Jutten (Eds.), Handbook of Blind Source Separation Independent Component Analysis and Applications, Academic Press, 2010. 
[9] N. Xu, X. Gao, B. Hong, X. Miao, S. Gao, F. Yang, BCI Competition 2003-Data Set IIb: Enhancing P300 Wave Detection Using ICA-Based Subspace Projections for BCI Applications, IEEE Trans. Biomed. Eng. 51 (6) (2004) 1067-1072.

[10] H. Serby, E. Yom-Tov, G. Inbar, An improved P300-based brain-computer interface, IEEE Trans. Neural Syst. Rehabil. Eng. 13 (1) (2005) 89-98.

[11] D. Mantini, M. Perrucci, S. Cugini, A. Ferretti, G. Romani, C. Del Gratta, Complete artifact removal for EEG recorded during continuous fMRI using independent component analysis, NeuroImage 34 (2) (2007) 598-607.

[12] Z. Koles, The quantitative extraction and topographic mapping of the abnormal components in the clinical EEG, Electroen. Clin. Neuro. 79 (6) (1991) 440-447.

[13] B. Blankertz, R. Tomioka, S. Lemm, M. Kawanabe, K.-R. Muller, Optimizing spatial filters for robust eeg single-trial analysis, IEEE Signal Process. Mag. 25 (1) (2008) $41-56$

[14] R. Fisher, The use of multiple measurements in taxonomic problems, Annals of Eugenics 7 (1936) 179-188.

[15] D. Model, M. Zibulevsky, Learning subject-specific spatial and temporal filters for single-trial EEG classification, NeuroImage 32 (4) (2006) 1631 $-1641$.

[16] R. Li, J. Principe, M. Bradley, V. Ferrari, A spatiotemporal filtering methodology for single-trial ERP component estimation, IEEE Trans. Biomed. Eng. 56 (1) (2009) 83 -92.

[17] R. Phlypo, N. Jrad, B. Rivet, M. Congedo, Common spatiotemporal pattern analysis, in: Proc. Int. Conf. Independent Component Analysis and Blind Source Separation (ICA), Saint-Malo, France, 2010, pp. 596-603.

[18] B. Rivet, A. Souloumiac, V. Attina, G. Gibert, xDAWN algorithm to enhance evoked potentials: Application to brain-computer interface, IEEE Trans. Biomed. Eng. 56 (8) (2009) 2035-2043.

[19] B. Rivet, A. Souloumiac, G. Gibert, V. Attina, "P300 speller" BrainComputer Interface: Enhancement of P300 evoked potential by spatial filters, in: Proc. European Signal Processing Conference (EUSIPCO), Lausanne, Switzerland, 2008.

[20] G. H. Golub, C. F. Van Loan, Matrix Computation, 3rd Edition, Johns Hopkins University Press, 1996.

[21] H. Krim, M. Viberg, Two decades of array signal processing research: the parametric approach, IEEE Signal Process. Mag. 13 (4) (1996) 67-94.

[22] A. Belouchrani, K. Abed-Meraim, J.-F. Cardoso, A blind source separation technique using second-order statistic, IEEE Trans. Signal Process. 45 (2) (1997) 434-444.

[23] J.-F. Cardoso, A. Souloumiac, Blind beamforming for non-gaussian signals, Radar and Signal Processing, IEE Proceedings F 140 (6) (1993) 362-370.

[24] A. Hyvarinen, Fast and Robust Fixed-Point Algorithms for Independent Component Analysis, IEEE Trans. Neural Netw. 10 (3) (1999) 626-634.

[25] G. H. Golub, H. Zha, The Canonical Correlations of Matrix Pairs and Their Numerical Computation, Tech. Rep. NA-92-13, Stanford University (1992).

[26] G. H. Golub, H. Zha, Perturbation analysis of the canonical correlations of matrix pairs, Linear Algebra Appl. 210 (1994) 3-28.

[27] H. Hotelling, Relations between two set of variables, Biometrika 28 (3-4) (1936) 321-377.

[28] G. Schalk, D. J. McFarland, T. Hinterberger, N. Birbaumer, J. R. Wolpaw, BCI2000: A General-Purpose Brain-Computer Interface (BCI) System, IEEE Trans. Biomed. Eng. 51 (6) (2004) 1034-1043.

[29] E. Donchin, K. Spencer, R. Wijesinghe, The mental prosthesis: assessing the speed of a P300-based brain-computer interface, IEEE Trans. Rehabil. Eng. 8 (2) (2000) 174-179.

[30] G. A. F. Seber, A Matrix Handbook for statisticians, Wiley, 2007. 